\title{
Brain-keratinocyte communication suffices for epidermal daily homeostasis
}

Thomas Mortimer" ${ }^{*}$, Valentina M. Zinna ${ }^{1}$, Carmelo Laudanna ${ }^{1}$, Paul Petrus ${ }^{2}$, Kevin B.

Koronowski², Jacob G. Smith ${ }^{2,4}$, Carolina M. Greco ${ }^{3}$, Elisa García-Lara ${ }^{1}$, Arun Kumar ${ }^{4}$, Mireia

Vaca-Dempere ${ }^{4}$, Oleg Deryagin ${ }^{4}$, Stephen Forrow ${ }^{1}$, Paolo Sassone-Corsi ${ }^{2}$, Patrick-Simon Welz ${ }^{6 *}$, Pura Muñoz-Cánoves ${ }^{4,5,7 *}$, Salvador Aznar Benitah, ${ }^{1,7^{*}}$

1. Institute for Research in Biomedicine (IRB Barcelona), The Barcelona Institute of Science and Technology (BIST), 08028, Barcelona, Spain.

2. Center for Epigenetics and Metabolism, U1233 INSERM, Department of Biological Chemistry, University of California, Irvine, CA 92697, USA.

3. Department of Biomedical Sciences, Humanitas University and Humanitas Research Hospital IRCCS, Via Manzoni 56, 20089, Rozzano (Milan), Italy.

4. Universitat Pompeu Fabra (UPF), Department of Experimental and Health Sciences (DCEXS) and CIBER on Neurodegenerative Diseases (CIBERNED), 08003, Barcelona, Spain.

5. Spanish National Center for Cardiovascular Research (CNIC), 28029, Madrid, Spain.

6. Hospital del Mar Medical Research Institute (IMIM), Cancer Research Programme, 08003, Barcelona, Spain.

7. Catalan Institution for Research and Advanced Studies (ICREA), 08010, Barcelona, Spain.

*Correspondence to: thomas.mortimer@irbbarcelona.org; pwelz@imim.es; pura.munoz@upf.edu; salvador.aznar-benitah@irbbarcelona.org

\section{$\underline{\text { Abstract }}$}

In mammals, an integrated network of molecular oscillators drives daily rhythms of tissuespecific homeostatic processes. This network is required for maintaining health and can be compromised by physiological aging, disease, and lifestyle choices. However, critical elements and properties of this network, such as key signaling nodes, the mechanisms of communication, and how physiological coherence is maintained, remain undefined. To dissect this system, we constructed a minimal clock network comprising only two nodes: the peripheral epidermal clock and the central brain clock. We observe that communication between the brain and epidermal clock is sufficient for wild-type core clock activity and specific homeostatic processes in the epidermis, yet full daily physiology requires input from other clock nodes. Moreover, our results show that the epidermal clock selectively suppresses or interprets systemic signals to ensure coherence of specific homeostatic processes, such as the cell cycle, identifying a previously-unrecognized gatekeeper role for this peripheral clock. This novel approach for dissecting a tissue's daily physiology reveals the specific rhythmic processes controlled by secondary tissues and niche components, opening the possibility of 
identifying the sources of age-related circadian disruption and potentially developing therapeutic interventions.

\section{Introduction}

Consistent and timely execution of homeostatic programs is essential for the long-term maintenance of organismal health (1). In mammals, many processes that maintain cellular, tissue, and systemic homeostasis exhibit a strict 24-hour periodicity that ensures their optimal alignment to diurnal changes in the internal and external environment (2). To drive this daily rhythmic physiology, mammals express a 24-hour molecular oscillator in almost all cell types that initiates time-of-day and tissue-specific homeostatic processes.

In mammals, the body's central clock is located in the hypothalamic suprachiasmatic nucleus (SCN) and promotes organism-wide coherence of daily physiology (3). Specifically, the SCN clock directly receives light signals through the retina, which enables it to phase-align with external (solar) time and to subsequently drive neural, hormonal, and behavioral rhythms that synchronize peripheral clocks in other tissues.

Complementing this regulatory mechanism, peripheral clocks also receive additional input directly from external cues (4-6) as well as internal signals from other peripheral clocks (710). Communication between the clocks likely relies on subsets of hormones, metabolites, and neural signals, yet the exact nature of these signals remains poorly defined (11). Importantly, incoherence introduced into this network of clocks through aging, disease, and/or lifestyle changes disrupts the daily homeostatic processes required for maintaining health $(7,12-15)$.

Identifying the specific function of each internode communication pathway is an essential first step towards understanding how this network maintains coherent daily physiology underlying health, and how to intervene when this is not maintained. To date, most studies have relied on disruption of putative signaling nodes (through genetic knockout, environmental interventions, or pharmacological inhibition) to dissect the systemic inputs that control daily tissue physiology (11). However, these approaches cannot isolate or allow interrogation of the individual clock-driven interactions between signaling nodes, in turn obscuring the specific aspects of daily physiology they drive, and the mechanisms by which they do so. To circumvent this, we reconstructed a minimal clock network in vivo constituting 
only the central clock (brain) (see accompanying article, (16)) and a single peripheral clock in the interfollicular epidermis. This approach allowed us to dissect the specific contributions of key signaling nodes within the network in driving a tissue's daily rhythmic physiology. Here we report that, although the central-peripheral communication was sufficient to drive wild-type core clock activity and specific homeostatic processes, complete rhythmic physiology was heavily reliant on peripheral-peripheral clock interactions. Unexpectedly, we found that the peripheral clock in epidermis can selectively suppress or integrate signals originating from the central clock. Thus, we identified a novel role for the peripheral clock as a gatekeeper in determining the influence of systemic signals on daily tissue homeostasis.

\section{$\underline{\text { Results }}$}

\section{Tissue-specific reconstitution of Bmall expression isolates communication between a peripheral (epidermal) clock and the central (brain) clock}

We set out to isolate (rather than to disrupt) the communication between a key signaling node and a target tissue, to understand how the node regulates the daily physiology of the tissue. We elected to focus first on signals generated by the central circadian clock in the brain, given its established role in controlling the body's network of tissue clocks. As the peripheral tissue receiving these signals, we chose to study the interfollicular epidermis of the skin, to exploit its extensively characterized daily physiology that becomes aberrantly rewired during $\operatorname{aging}(5,14,17)$.

To isolate brain:epidermal clock communication, we used a mouse model in which tissuespecific expression of a Cre-recombinase reconstitutes the endogenous expression of the indispensable core clock component BMAL1 - and thus clock activity-in an otherwise clock-deficient mouse (herein, Bmal1-StopFL mice) (5). Previously, we showed that keratin 14-Cre-driven reconstitution (RE) of BMAL1 expression in Bmal1-StopFL mice (herein, epi-RE mice) leads to clock activity and a limited daily physiology specifically in the interfollicular epidermis - e.g., in a tissue-autonomous manner (5). To determine which daily physiology functions only rely on receiving brain clock signals (and do not need clock communication), we generated a Bmal1-StopFL mouse model that expressed Crerecombinase from the synaptotagmin 10 promoter (Syt10-Cre), which is expressed 
specifically in the brain (with high expression in the SCN) (18), to give brain-reconstituted (brain-RE) mice (Fig. 1A). Finally, we crossed epi-RE mice with the Syt10-Cre mice, to produce a mouse line with reconstituted clocks in both the epidermis and the brain/SCN, giving a double-reconstituted (RE/RE) mouse model for studying brain:epidermal clock communication (Fig. 1A). Western blot analysis confirmed that Syt10-Cre-driven BMAL1 expression was restricted to the brain (Fig. 1B), and immunofluorescence revealed that BMAL1 expression was restored in the SCN and other neuronal regions in which the Syt10Cre is expressed (Fig. 1C and fig S1, A and B). Importantly, BMAL1 expression in the SCN was sufficient to drive daily cycles of activity, feeding and metabolism (see accompanying article, (16)), indicating that the SCN clock was functioning correctly.

Peripheral clocks, including those in skin, require communication with the brain clock for full entrainment to external conditions $(4,19)$. To identify whether brain:epidermal clock communication was restored in RE/RE mice, we characterized whether the reconstituted brain clock entrained the epidermal clock. For this, we first obtained the diurnal epidermal transcriptome from epi-RE, brain-RE, RE/RE, and WT mice (eight-week-old male mice, under a 12h:12h light/dark [LD] cycle). Strikingly, in epidermis from RE/RE mice, genes of all core components of the epidermal clock, as well as several key clock-controlled genes, including Dbp, Tef and $P d x k$ (20-22), oscillated with amplitudes, phases, and periods that were indistinguishable from WT mice (Fig. 1, D and E). In contrast, and as previously described, oscillation patterns of core clock genes and clock-controlled genes in epidermis from epi-RE or brain-RE mice were dampened or absent, respectively, as compared to those of WT mice (Fig. 1, D and E) $(5,6)$. Thus, we concluded that that our model reconstituted known communication pathways between brain and peripheral clocks, making it appropriate to elucidate the degree to which the brain clock controls a tissues daily epidermal physiology.

\section{Intact daily epidermal physiology requires communication with other peripheral tissues}

Daily epidermal physiology comprises a rhythmic execution of the cell cycle, DNA repair, cell differentiation, and metabolic pathways, which together maintain tissue integrity and functionality $(14,23,24)$. Underlying this daily physiology are diurnal oscillations in the expression of thousands of transcripts, which endow rhythmicity to homeostatic processes. 
To determine the role of brain clock signals in driving daily epidermal physiology, we compared the diurnal epidermis transcriptomes of epi-RE, brain-RE, or RE/RE mice to that of WT mice (Fig. 2A and fig. S2, A and B). Strikingly, and in sharp contrast to the indistinguishable gene oscillations of their core clock machinery components (see Fig. 1, E and F), the diurnal transcriptome of RE/RE epidermis showed only a 34\% (1060 genes) overlap with that of WT (Fig. 2B). Nonetheless, this overlap was substantially larger than that of brain-RE and WT (20.2\%, 628 genes) or of epi-RE and WT (11.6\%, 363 genes) (Fig. 2B). Moreover, 20.9\% (651 genes) of the WT diurnal transcriptome relied specifically on communication between the epidermal and brain clocks for rhythmic expression (Fig. 2, B and C), demonstrating an important role for brain:epidermal clock communication in driving diurnal transcriptional programs. These communication-dependent transcripts showed similar phases and amplitudes in epidermis from RE/RE and WT mice (Fig. 2, D and E), suggesting that brain:epidermal clock communication alone was sufficient to drive their physiological rhythmic expression. Unexpectedly, a further 51\% (1588 genes) of WT rhythmic transcripts oscillated uniquely in WT epidermis and not in any other condition (Fig. 2, B and C). This class of rhythmic transcripts represented those that are reliant on the wider clock network that is present in WT mice but not in RE/RE mice-in other words, they require communication between the epidermal clock and other peripheral clocks (or local niche clocks). This indicates a key role for peripheral/niche clock:epidermal clock communication (herein, peripheral:epidermal) in regulating the diurnal transcriptome of the epidermis. We identified similar patterns using an independent algorithm (BIO_CYCLE) to define the diurnal transcriptomes of each condition, thus confirming our observations (fig. $\mathrm{S} 3, \mathrm{~A}, \mathrm{~B}$, and E).

We then dissected the specific homeostatic processes constituting daily epidermal physiology that were regulated by either brain:epidermal or peripheral:epidermal clock communication. The gene set requiring brain:epidermal clock communication for its daily rhythmicity in expression - that is, the set with overlap only between WT and RE/RE mice (651 genes) was enriched for terms relating to cell cycle, circadian rhythm, and vesicle transport (Fig. 2F and fig. S2C). In contrast, the gene set that required peripheral:epidermal clock communication (e.g., present in WT only; 1589 genes) were enriched for genes involved in RNA processing, protein processing, and cellular differentiation (Fig. $2 \mathrm{~F}$ and fig. S2C). 
Further confirming this functional demarcation, gene ontology analysis of the whole diurnal transcriptome from each condition showed similar patterns (Fig. $2 \mathrm{H}$ and fig. S2D). In particular, we observed functional distinctions of transcript enrichment in the various diurnal transcriptomes:

i) only in WT epidermis: transcripts for regulators of mitochondrial organization (a key process in keratinocyte differentiation; $(25,26))$ and for components of the Wnt and Notch pathways (fig. S2D). Notably, the latter included the Wnt ligand receptor Frizzled 2 (FZD2) and Notch ligand Delta-like 1 (Dll1), indicating possible mechanisms by which rhythmic differentiation is achieved in WT epidermis (fig. S2E);

ii) only in epidermis from WT mice and RE/RE mice: transcripts for vesicular trafficking (fig. S2D), and for proteins associated with later stages of the cell cycle (G2/M, cytokinesis) (Fig. 2G); including CDK1, cyclin B1, and cyclin B2 (which are essential regulators of the G2/M transition) (Fig. 2H);

iii) in epidermis from WT, RE/RE, epi-RE, and brain-RE mice: transcripts for pathways related to early phases of the cell cycle (e.g., G0/G1 transition and DNA replication/S phase) (Fig. 2G).

Importantly, cell cycle analysis by flow cytometry confirmed that the late cell cycle was indeed rhythmic in WT and RE/RE epidermis, but not in brain-RE or epi-RE epidermis (Fig. 2I). Overall, this suggested that while rhythmic initiation of the cell cycle can be driven autonomously by the epidermal clock or by systemic signals, correct temporal gating of the full cell cycle relies on brain:epidermal clock communication. We concluded that the brain clock contributes significantly towards driving rhythmicity of specific homeostatic processes in the epidermis. However, signals originating from other clock nodes are indispensable for a full program of daily epidermal physiology.

The core clock machinery cooperates with tissue-specific transcription factors to drive diurnal transcriptional rhythms (27). As such, we hypothesized that the brain and peripheral clock signals may synergize with the epidermal clock by regulating specific transcription factors that shape its output. Supporting our hypothesis, the promoters of rhythmically expressed genes regulated by peripheral:epidermal clock communication (e.g., only in WT 
epidermis; 1589 genes) or brain:epidermal clock communication (e.g., only in WT epidermis and RE/RE epidermis; 651 genes) were enriched for the binding sites of distinct sets of transcription factors (Fig. 2J). Interestingly, the nutrient responsive transcription factors SREBP2a and -1 (28) were enriched in the gene set driven by peripheral:epidermal clock communication (Fig. 2J), suggesting that peripheral clock regulation of systemic nutrient homeostasis may act as an input that controls epidermal clock output. In contrast, transcription factors related to cellular proliferation (RREB1 [28] and RUNX2 [29]) and epidermal barrier formation (KLF4 [30]) were enriched in the gene set driven by brain:epidermal clock communication (Fig. 2J), in line with the homeostatic processes that rely on this communication pathway.

\section{The epidermal clock selectively gates brain clock signals}

In mice, epidermal DNA replication peaks during night, thereby avoiding exposing the vulnerable DNA to the daytime peak of DNA-damaging oxidative conditions $(2,23,32,33)$. Our data indicates that both local and systemic mechanisms are able to independently drive this key aspect of daily epidermal physiology (Fig. 2H). Representing potential targets of this redundant regulation, 67 transcripts shared rhythmicity in epidermis from all mice models (e.g., WT, epi-RE, brain-RE, and RE/RE mice) (Fig. 3A). Importantly, comparison with existing data from Welz et al. (2019) showed that only 1 of the 67 transcripts with shared rhythmicity oscillated in the epidermis of whole-body Bmal1-knockout (KO) mice (Fig. 3B), confirming the reliance of the other transcripts on local or brain clock activity for oscillatory expression. As expected, shared rhythmicity transcripts were highly enriched for genes related to G0/G1 transition and DNA replication (Fig. 3C). Unexpectedly, while transcript expression peaked at nighttime between ZT14 and ZT16 in WT, RE/RE, and epi-RE mice, it peaked at the onset of light hours (between ZT0 and ZT2) when only the brain clock was present (brain-RE), thus showing an antiphasic expression pattern (Fig. 3D). Thus, when working alone, the brain clock drove a peak in DNA replication that coincided with the onset of light but also with the maximum DNA damaging conditions in the epidermis. Importantly, however, this peak was corrected by the presence of the local epidermal clock. Genes exhibiting antiphasic expression included indispensable components of the DNA replication machinery, such as those encoding MCM2-4, GINS2, PCNA, and POLE, and core regulators 
of the early cell cycle CDK2, CCND1, D2, E1, and E2 (Fig. 3E). The brain-driven antiphasic expression was observed only for genes of the early cell cycle, as other transcripts that were rhythmic in both WT and brain-RE epidermis showed similar phases (Fig. 3F). Thus, we describe a previously unidentified function of epidermal clock whereby it is essential for gating signals originating from the brain clock, which would otherwise compromise the coherence between daily rhythms in DNA replication and the nighttime reduction of DNAdamaging conditions.

\section{Brain clock communication ensures robust daily epidermal physiology in the absence of environmental cues}

Robustness against short-term variations in environmental conditions is essential for maintaining a consistent daily physiology. Signals from the autonomously oscillating brain clock are key for ensuring correctly timed execution of daily physiology even when environmental cues are acutely disrupted or absent $(4,34)$. However, it is unknown whether the brain clock alone is sufficient for this stabilizing function, or if cooperation with other elements of the clock network are also required. To define the sufficiency of brain clock communication in endowing stability to daily physiology in peripheral tissues, we characterized the epidermal circadian transcriptome in WT, RE/RE, epi-RE, and brain-RE mice (all 8-week-old females) in the absence of normal light/dark (LD) cycles (i.e., after one week of constant darkness). The number of transcripts of the epidermal circadian transcriptome in dark/dark (DD) conditions relative to LD conditions remained largely unchanged in WT mice (3115 in LD vs 2669 in DD) and RE/RE mice (1862 in LD vs 1896 in DD) but was substantially reduced in epi-RE mice (756 in LD vs 256 in DD) and brainRE mice (1662 in LD vs 579 in DD) (Fig. 4A). Strikingly, core clock genes oscillated identically in epidermis from WT and RE/RE mice in DD conditions (Fig. 4B), demonstrating that communication with the brain clock alone is sufficient to ensure a stable epidermal clock entrainment in the absence of light. Moreover, the rhythmicity of the clock output genes $D b p$, Tef, and $P d x k$ was indistinguishable in epidermis from WT mice and $\mathrm{RE} / \mathrm{RE}$ mice, further confirming the presence of a WT-like clock activity in RE/RE epidermis in the absence of light (fig. S4C). In contrast, the core clock genes lacked rhythmicity in epidermis from brain-RE or epi-RE mice (Fig. 4B). Our observation that the DD condition 
led to loss of core clock activity and decreased circadian transcriptome in epi-RE epidermis is in line with the observation that autonomous epidermal clock entrainment is driven by light cycles (5).

In the DD condition, $40.4 \%$ of the WT circadian transcriptome (1078 genes) was shared with RE/RE epidermis (Fig. 4C). Of these, 35.2\% (938 genes) specifically required brain:epidermal clock communication for rhythmicity (Fig. 4C), mirroring the proportions observed under the LD condition (see Fig. 2B). These shared rhythmic transcripts showed a similar phase distribution and a small reduction in amplitude in RE/RE epidermis with respect to WT epidermis (Fig. 4, D and E). Furthermore, 55.2\% of WT circadian transcripts (1473 genes) oscillated only in WT epidermis under DD conditions (Fig. 4C). These results further support our conclusion that a substantial contribution of peripheral clock input is required to ensure a physiological circadian transcriptome and function. Further, as for LD conditions, defining the diurnal transcriptomes of each condition using an independent algorithm (BIO_CYCLE) found similar patterns (fig. S3C, D and F). Thus, we concluded that brain:epidermal clock communication that drives epidermal clock entrainment and output was maintained even in the absence of light; as such, it represents a stable communication pathway for ensuring robust daily epidermal physiology.

Under DD conditions, the brain clock continued to output dichotomous signals regulating epidermal cell cycle rhythmicity. Genes with shared rhythmicity in WT, RE/RE, and brainRE epidermis (109 genes) were highly enriched in categories related to the early cell cycle (fig. S4D) but were expressed in an opposite phase in brain-RE epidermis (fig. S4E). Once again, this included genes encoding core regulators of the G0/G1 transition, such as CDK2 and E2F1, and key components of the DNA replication machinery, such as MCM2-6, POLE, and PCNA (Fig. 4, G and H, and fig. S4F). In contrast, rhythmicity in later phases of the cell cycle (G2/M transition and cytokinesis) was observed only in epidermis from WT and RE/RE mice (Fig. 4, G and H). Similar to LD conditions, the core regulators of the G2/M transitionCDK1, CCNB1, and CCNB2 - were rhythmically expressed only in epidermis from RE/RE and WT mice in DD conditions (Fig. 4H). As expected, no cell cycle rhythmicity was observed at the transcriptomic level in epi-RE in the absence of light (Fig. 4G) (5). Together, these results demonstrated that brain clock signals regulating the epidermal cell cycle are 
circadian in nature, and confirmed that the local epidermal clock must both integrate and suppress distinct systemic signals to ensure correct daily rhythms of the cell cycle.

\section{$\underline{\text { Discussion }}$}

We have comprehensively dissected for the first time the role of a specific signaling node in defining and coordinating a distal tissue's daily physiology in vivo. By isolating the brain:epidermal clock communication axis, we show that this connection is sufficient to drive epidermal clock entrainment as well as for specific rhythmic homeostatic processes, yet, importantly, it is insufficient to ensure full daily physiology. In particular, the brain:epidermal clock communication alone suffices for the rhythmicity underlying the cell cycle and vesicular trafficking, but not for rhythmicity in RNA processing, protein processing, or correct expression of differentiation signals. In other words, these additional functions require input from other peripheral clocks, and therefore communication with niche cells and other peripheral tissues is essential for attaining a complete daily physiology. In this sense, growing evidence suggests that peripheral clock communication is a conserved motif across tissue types and organ systems (7-11). For instance, recent studies suggest that extensive communication may exist between the clocks of metabolic tissues, such as the liver and skeletal muscle, as well as between distinct cell types within individual tissues $(7,10)$. Future studies will be necessary to determine the exact identity of the peripheral tissues/niche components needed for intact epidermal daily physiology.

Together, our study indicates that peripheral tissue clocks are heavily reliant on input from other peripheral clocks, which in turn cooperate with the local core clock machinery to execute full daily physiology. This finding supports the proposed federated structure of the clock network (11), in which each clock receives and consolidates diverse inputs from the environment, the central clock of the SCN, and other peripheral clocks to determine its daily physiology. This model contrasts with the traditional hierarchical model of the clock network, in which signals from the central brain clock define the activity of subservient peripheral oscillators found in other body tissues (35). Although our double-reconstitution models cannot rule out that the signals emanating from other peripheral tissues still rely on the entrainment by the brain, our data suggest that a large proportion of the rhythmic homeostatic processes nevertheless depend on input from the broader clock network. 
Unexpectedly, we identified the epidermal cell cycle as a paradigm for cooperation and conflict between the brain and peripheral clocks. When unchecked by the local epidermal clock, brain clock signals drive a morning peak in execution of early cell cycle phases (G0/G1 transition and $\mathrm{S}$ phase) that coincides with a daily maximum in DNA-damaging oxidizing conditions - a profile antiphasic to that of wild-type epidermis. However, the presence of the local clock suppresses this general brain signal and corrects the phase of DNA replication, which in turn is likely to minimize DNA damage. This interaction suggests that the epidermal clock has an unexpected gatekeeper role, whereby it selectively represses systemic signals that would otherwise compromise the coherence of homeostatic processes. Strikingly, and in direct contrast with the early cell cycle, we also show that brain:epidermal clock communication is sufficient to ensure physiological rhythmicity in execution of late cell cycle stages (G2/M transition and cytokinesis). Given these opposing dual roles of the brain clock, we propose that the brain exerts the essential task of entraining the rest of the body to changes in light; however, the signals emanating from the brain clock are not complex enough to modulate the hundreds of functions each tissue must perform according to its biology. In turn, peripheral clocks selectively interpret and repress systemic signals originating from the brain clock (and potentially those from other peripheral clocks), depending on whether the systemic conditions they reflect are relevant to the execution of that tissue's daily physiology (e.g., epidermal DNA replication and oxidative damage). Peripheral clocks respond differentially to entraining signals, such as feeding and light, implying tissue-specific mechanisms that recognize or even suppress such signals $(10,36)$. Interestingly, recent evidence suggests that the liver and muscle clocks are able to buffer the response of specific peripheral clocks to feeding-related signals $(9,10)$, highlighting a potential role for inter-peripheral clock communication in regulating how a tissue responds to brain clock signals. Here, we expand this concept, implicating the local clock as both a transducer and repressor of systemic signals.

We believe that our findings can also have significant implications for human health. Notably, distinct cell types and tissues, including epidermal stem cells, muscle satellite cells, liver hepatocytes, adipose tissue, and the prefrontal cortex, experience aberrant rewiring of their daily physiology during aging that allows them to cope with daily age-related stress (13, $14,37,38)$. However, a subset of core functions remains rhythmic in the aged peripheral 
tissues which interestingly coincide with those we have identified as tissue-autonomous (i.e. present in epi-RE mice and WT mice). By comparing the aspects of a tissue's daily physiology that decay with age to those requiring communication with specific signaling nodes, we are identifying for the first time nodes that are likely to be important in a tissue's aging process. Such knowledge will provide invaluable insight into defining targets for future anti-aging or rejuvenation therapies.

\section{$\underline{\text { References }}$}

1. C. López-Otín, G. Kroemer, Hallmarks of Health. Cell. 184 (2021), pp. 33-63.

2. S. A. Benitah, P. S. Welz, Circadian Regulation of Adult Stem Cell Homeostasis and Aging. Cell Stem Cell. 26 (2020), pp. 817-831.

3. M. H. Hastings, E. S. Maywood, M. Brancaccio, Generation of circadian rhythms in the suprachiasmatic nucleus. Nat. Rev. Neurosci. 19 (2018), pp. 453-469.

4. J. Husse, A. Leliavski, A. H. Tsang, H. Oster, G. Eichele, The light-dark cycle controls peripheral rhythmicity in mice with a genetically ablated suprachiasmatic nucleus clock. FASEB J. 28, 4950-4960 (2014).

5. P. S. Welz, V. M. Zinna, A. Symeonidi, K. B. Koronowski, K. Kinouchi, J. G. Smith, I. M. Guillén, A. Castellanos, G. Crainiciuc, N. Prats, J. M. Caballero, A. Hidalgo, P. Sassone-Corsi, S. A. Benitah, BMAL1-Driven Tissue Clocks Respond Independently to Light to Maintain Homeostasis. Cell. 177, 1436-1447.e12 (2019).

6. K. B. Koronowski, K. Kinouchi, P. S. Welz, J. G. Smith, V. M. Zinna, J. Shi, M. Samad, S. Chen, C. N. Magnan, J. M. Kinchen, W. Li, P. Baldi, S. A. Benitah, P. Sassone-Corsi, Defining the Independence of the Liver Circadian Clock. Cell. 177, 1448-1462.e14 (2019).

7. K. A. Dyar, D. Lutter, A. Artati, N. J. Ceglia, Y. Liu, D. Armenta, M. Jastroch, S. Schneider, S. de Mateo, M. Cervantes, S. Abbondante, P. Tognini, R. Orozco-Solis, K. Kinouchi, C. Wang, R. Swerdloff, S. Nadeef, S. Masri, P. Magistretti, V. Orlando, E. Borrelli, N. H. Uhlenhaut, P. Baldi, J. Adamski, M. H. Tschöp, K. Eckel-Mahan, P. Sassone-Corsi, Atlas of Circadian Metabolism Reveals Systemwide Coordination and Communication between Clocks. Cell. 174, 1571-1585.e11 (2018).

8. D. Guan, Y. Xiong, T. M. Trinh, Y. Xiao, W. Hu, C. Jiang, P. Dierickx, C. Jang, J. D. Rabinowitz, M. A. Lazar, The hepatocyte clock and feeding control chronophysiology of multiple liver cell types. Science (80-. ). 369, 1388-1395 (2020).

9. C. M. Greco, K. B. Koronowski, J. G. Smith, J. Shi, P. Kunderfranco, R. Carriero, S. Chen, M. Samad, P. S. Welz, V. M. Zinna, T. Mortimer, S. K. Chun, K. Shimaji, T. Sato, P. Petrus, A. Kumar, M. Vaca-Dempere, O. Deryagian, C. Van, J. M. M. Kuhn, D. Lutter, M. M. Seldin, S. Masri, W. Li, P. Baldi, K. A. Dyar, P. Muñoz-Cánoves, 
S. A. Benitah, P. Sassone-Corsi, Integration of feeding behavior by the liver circadian clock reveals network dependency of metabolic rhythms. Sci. Adv. (2021), doi:10.1126/sciadv.abi7828.

10. G. Manella, E. Sabath, R. Aviram, V. Dandavate, S. Ezagouri, M. Golik, Y. Adamovich, G. Asher, The liver-clock coordinates rhythmicity of peripheral tissues in response to feeding. Nat. Metab. (2021), doi:10.1038/s42255-021-00395-7.

11. L. V. M. de Assis, H. Oster, The circadian clock and metabolic homeostasis: entangled networks. Cell. Mol. Life Sci. 78 (2021), , doi:10.1007/s00018-021-038002.

12. S. Masri, T. Papagiannakopoulos, K. Kinouchi, Y. Liu, M. Cervantes, P. Baldi, T. Jacks, P. Sassone-Corsi, Lung Adenocarcinoma Distally Rewires Hepatic Circadian Homeostasis. Cell. 165, 896-909 (2016).

13. S. Sato, G. Solanas, F. O. Peixoto, L. Bee, A. Symeonidi, M. S. Schmidt, C. Brenner, S. Masri, S. A. Benitah, P. Sassone-Corsi, Circadian Reprogramming in the Liver Identifies Metabolic Pathways of Aging. Cell (2017), doi:10.1016/j.cell.2017.07.042.

14. G. Solanas, F. O. Peixoto, E. Perdiguero, M. Jardí, V. Ruiz-Bonilla, D. Datta, A. Symeonidi, A. Castellanos, P. S. Welz, J. M. Caballero, P. Sassone-Corsi, P. MuñozCánoves, S. A. Benitah, Aged Stem Cells Reprogram Their Daily Rhythmic Functions to Adapt to Stress. Cell (2017), doi:10.1016/j.cell.2017.07.035.

15. N. Schaum, B. Lehallier, O. Hahn, R. Pálovics, S. Hosseinzadeh, S. E. Lee, R. Sit, D. P. Lee, P. M. Losada, M. E. Zardeneta, T. Fehlmann, J. T. Webber, A. McGeever, K. Calcuttawala, H. Zhang, D. Berdnik, V. Mathur, W. Tan, A. Zee, M. Tan, N. Almanzar, J. Antony, A. S. Baghel, I. Bakerman, I. Bansal, B. A. Barres, P. A. Beachy, D. Berdnik, B. Bilen, D. Brownfield, C. Cain, C. K. F. Chan, M. B. Chen, M. F. Clarke, S. D. Conley, S. Darmanis, A. Demers, K. Demir, A. de Morree, T. Divita, H. du Bois, H. Ebadi, F. H. Espinoza, M. Fish, Q. Gan, B. M. George, A. Gillich, R. Gòmez-Sjöberg, F. Green, G. Genetiano, X. Gu, G. S. Gulati, O. Hahn, M. S. Haney, Y. Hang, L. Harris, M. He, S. Hosseinzadeh, A. Huang, K. C. Huang, T. Iram, T. Isobe, F. Ives, R. Jones, K. S. Kao, J. Karkanias, G. Karnam, A. Keller, A. M. Kershner, N. Khoury, S. K. Kim, B. M. Kiss, W. Kong, M. A. Krasnow, M. E. Kumar, C. S. Kuo, J. Y. Lam, D. P. Lee, S. E. Lee, B. Lehallier, O. Leventhal, G. Li, Q. Li, L. Liu, A. Lo, W. J. Lu, M. F. Lugo-Fagundo, A. Manjunath, A. P. May, A. Maynard, A. McGeever, M. McKay, M. W. McNerney, B. Merrill, R. J. Metzger, M. Mignardi, D. Min, A. N. Nabhan, N. F. Neff, K. M. Ng, P. K. Nguyen, J. Noh, R. Nusse, R. Pálovics, R. Patkar, W. C. Peng, L. Penland, A. O. Pisco, K. Pollard, R. Puccinelli, Z. Qi, S. R. Quake, T. A. Rando, E. J. Rulifson, N. Schaum, J. M. Segal, S. S. Sikandar, R. Sinha, R. V. Sit, J. Sonnenburg, D. Staehli, K. Szade, M. Tan, W. Tan, C. Tato, K. Tellez, L. B. T. Dulgeroff, K. J. Travaglini, C. Tropini, M. Tsui, L. Waldburger, B. M. Wang, L. J. van Weele, K. Weinberg, I. L. Weissman, M. N. Wosczyna, S. M. Wu, T. Wyss-Coray, J. Xiang, S. Xue, K. A. Yamauchi, A. C. Yang, L. P. Yerra, J. Youngyunpipatkul, B. Yu, F. Zanini, M. E. Zardeneta, A. Zee, C. Zhao, F. Zhang, H. Zhang, M. J. Zhang, L. Zhou, J. Zou, A. O. Pisco, J.

Karkanias, N. F. Neff, A. Keller, S. Darmanis, S. R. Quake, T. Wyss-Coray, Ageing 
hallmarks exhibit organ-specific temporal signatures. Nature (2020), doi:10.1038/s41586-020-2499-y.

16. P. Petrus, J. G. Smith, K. B. Koronowski, S. Chen, T. Sato, C. M. Greco, T. Mortimer, P.-S. Welz, V. M. Zinna, K. Shimaji, M. Cervantes, P. Baldi, P. M. Canoves, P. Sassone-Corsi, S. A. Benitah, bioRxiv, in press, doi:10.1101/2022.01.24.477514.

17. P. Janich, K. Toufighi, G. Solanas, N. M. Luis, S. Minkwitz, L. Serrano, B. Lehner, S. A. Benitah, Human epidermal stem cell function is regulated by circadian oscillations. Cell Stem Cell (2013), doi:10.1016/j.stem.2013.09.004.

18. J. Husse, X. Zhou, A. Shostak, H. Oster, G. Eichele, Synaptotagmin10-cre, a driver to disrupt clock genes in the SCN. J. Biol. Rhythms. 26, 379-389 (2011).

19. M. Tanioka, H. Yamada, M. Doi, H. Bando, Y. Yamaguchi, C. Nishigori, H. Okamura, Molecular clocks in mouse skin. J. Invest. Dermatol. (2009), doi:10.1038/jid.2008.345.

20. P. Fonjallaz, V. Ossipow, G. Wanner, U. Schibler, The two PAR leucine zipper proteins, TEF and DBP, display similar circadian and tissue-specific expression, but have different target promoter preferences. EMBO J. (1996), doi:10.1002/j.14602075.1996.tb00365.x.

21. F. Gachon, P. Fonjallaz, F. Damiola, P. Gos, T. Kodama, J. Zakany, D. Duboule, B. Petit, M. Tafti, U. Schibler, The loss of circadian PAR bZip transcription factors results in epilepsy. Genes Dev. 18, 1397 (2004).

22. J. A. Ripperger, L. P. Shearman, S. M. Reppert, U. Schibler, CLOCK, an essential pacemaker component, controls expression of the circadian transcription factor DBP. Genes Dev. (2000), doi:10.1101/gad.14.6.679.

23. M. Geyfman, V. Kumar, Q. Liu, R. Ruiz, W. Gordon, F. Espitia, E. Cam, S. E. Millar, P. Smyth, A. Ihler, J. S. Takahashi, B. Andersen, Brain and muscle Arnt-like protein-1 (BMAL1) controls circadian cell proliferation and susceptibility to UVBinduced DNA damage in the epidermis. Proc. Natl. Acad. Sci. U. S. A. 109, 1175811763 (2012).

24. P. Janich, Q. J. Meng, S. A. Benitah, Circadian control of tissue homeostasis and adult stem cells. Curr. Opin. Cell Biol. (2014), , doi:10.1016/j.ceb.2014.06.010.

25. R. B. Hamanaka, A. Glasauer, P. Hoover, S. Yang, H. Blatt, A. R. Mullen, S. Getsios, C. J. Gottardi, R. J. DeBerardinis, R. M. Lavker, N. S. Chandel, Mitochondrial reactive oxygen species promote epidermal differentiation and hair follicle development. Sci. Signal. 6 (2013), doi:10.1126/scisignal.2003638.

26. A. Sreedhar, L. Aguilera-Aguirre, K. K. Singh, Mitochondria in skin health, aging, and disease. Cell Death Dis. 11 (2020), , doi:10.1038/s41419-020-2649-z.

27. J. R. Beytebiere, A. J. Trott, B. J. Greenwell, C. A. Osborne, H. Vitet, J. Spence, S. H. Yoo, Z. Chen, J. S. Takahashi, N. Ghaffari, J. S. Menet, Tissue-specific BMAL1 cistromes reveal that rhythmic transcription is associated with rhythmic enhancer- 
enhancer interactions. Genes Dev. 33, 294-309 (2019).

28. R. A. DeBose-Boyd, J. Ye, SREBPs in Lipid Metabolism, Insulin Signaling, and Beyond. Trends Biochem. Sci. (2018), , doi:10.1016/j.tibs.2018.01.005.

29. Y. N. Deng, Z. Xia, P. Zhang, S. Ejaz, S. Liang, Transcription factor RREB1: From target genes towards biological functions. Int. J. Biol. Sci. 16, 1463-1473 (2020).

30. D. J. Glotzer, E. Zelzer, B. R. Olsen, Impaired skin and hair follicle development in Runx2 deficient mice. Dev. Biol. 315, 459-473 (2008).

31. J. A. Segre, C. Bauer, E. Fuchs, Klf4 is a transcription factor required for establishing the barrier function of the skin. Nat. Genet. 22, 356-360 (1999).

32. S. Gaddameedhi, C. P. Selby, W. K. Kaufmann, R. C. Smart, A. Sancar, Control of skin cancer by the circadian rhythm. Proc. Natl. Acad. Sci. U. S. A. 108, 1879018795 (2011).

33. C. Stringari, H. Wang, M. Geyfman, V. Crosignani, V. Kumar, J. S. Takahashi, B. Andersen, E. Gratton, In Vivo Single-Cell Detection of Metabolic Oscillations in Stem Cells. Cell Rep. (2015), doi:10.1016/j.celrep.2014.12.007.

34. R. M. Buijs, M. A. Guzmán Ruiz, R. Méndez Hernández, B. Rodríguez Cortés, The suprachiasmatic nucleus; a responsive clock regulating homeostasis by daily changing the setpoints of physiological parameters. Auton. Neurosci. Basic Clin. 218 (2019), pp. 43-50.

35. C. Dibner, U. Schibler, U. Albrecht, The mammalian circadian timing system: Organization and coordination of central and peripheral clocks. Annu. Rev. Physiol. 72 (2009), pp. 517-549.

36. F. Damiola, N. Le Minli, N. Preitner, B. Kornmann, F. Fleury-Olela, U. Schibler, Restricted feeding uncouples circadian oscillators in peripheral tissues from the central pacemaker in the suprachiasmatic nucleus. Genes Dev. 14, 2950-2961 (2000).

37. G. M. Sutton, A. A. Ptitsyn, Z. E. Floyd, G. Yu, X. Wu, K. Hamel, F. S. Shah, A. Centanni, K. Eilertsen, I. Kheterpal, S. Newman, C. Leonardi, M. A. Freitas, B. A. Bunnell, J. M. Gimble, Biological aging alters circadian mechanisms in murine adipose tissue depots. Age (Omaha). 35, 533-547 (2013).

38. C. Y. Chen, R. W. Logan, T. Ma, D. A. Lewis, G. C. Tseng, E. Sibille, C. A. McClung, Effects of aging on circadian patterns of gene expression in the human prefrontal cortex. Proc. Natl. Acad. Sci. U. S. A. 113, 206-211 (2016).

39. N. Percie du Sert, V. Hurst, A. Ahluwalia, S. Alam, M. T. Avey, M. Baker, W. J. Browne, A. Clark, I. C. Cuthill, U. Dirnagl, M. Emerson, P. Garner, S. T. Holgate, D. W. Howells, N. A. Karp, S. E. Lazic, K. Lidster, C. J. MacCallum, M. Macleod, E. J. Pearl, O. H. Petersen, F. Rawle, P. Reynolds, K. Rooney, E. S. Sena, S. D. Silberberg, T. Steckler, H. Würbel, The ARRIVE guidelines 2.0: Updated guidelines for reporting animal research*. J. Cereb. Blood Flow Metab. 40, 1769-1777 (2020). 
40. H. R. Dassule, P. Lewis, M. Bei, R. Maas, A. P. McMahon, Sonic hedgehog regulates growth and morphogenesis of the tooth. Development (2000), doi:10.1242/dev.127.22.4775.

41. A. M. Bolger, M. Lohse, B. Usadel, Trimmomatic: A flexible trimmer for Illumina sequence data. Bioinformatics (2014), doi:10.1093/bioinformatics/btu170.

42. D. Kim, J. M. Paggi, C. Park, C. Bennett, S. L. Salzberg, Graph-based genome alignment and genotyping with HISAT2 and HISAT-genotype. Nat. Biotechnol. (2019), doi:10.1038/s41587-019-0201-4.

43. H. Li, B. Handsaker, A. Wysoker, T. Fennell, J. Ruan, N. Homer, G. Marth, G. Abecasis, R. Durbin, 1000 Genome Project Data Processing 1000 Genome Project Data Processing Subgroup, The Sequence Alignment/Map format and SAMtools. Bioinformatics. 25, 2078-9 (2009).

44. Y. Liao, G. K. Smyth, W. Shi, FeatureCounts: An efficient general purpose program for assigning sequence reads to genomic features. Bioinformatics (2014), doi:10.1093/bioinformatics/btt656.

45. M. D. Robinson, D. J. McCarthy, G. K. Smyth, edgeR: a Bioconductor package for differential expression analysis of digital gene expression data. Bioinformatics. 26, 139 (2010).

46. M. E. Hughes, J. B. Hogenesch, K. Kornacker, JTK_CYCLE: an efficient nonparametric algorithm for detecting rhythmic components in genome-scale data sets. J. Biol. Rhythms. 25, 372-80 (2010).

47. F. Agostinelli, N. Ceglia, B. Shahbaba, P. Sassone-Corsi, P. Baldi, What time is it? Deep learning approaches for circadian rhythms. Bioinformatics. 32, i8-i17 (2016).

48. E. Y. Chen, C. M. Tan, Y. Kou, Q. Duan, Z. Wang, G. V. Meirelles, N. R. Clark, A. Ma'ayan, Enrichr: Interactive and collaborative HTML5 gene list enrichment analysis tool. BMC Bioinformatics (2013), doi:10.1186/1471-2105-14-128.

49. F. Zambelli, G. Pesole, G. Pavesi, Pscan: Finding over-represented transcription factor binding site motifs in sequences from co-regulated or co-expressed genes. Nucleic Acids Res. (2009), doi:10.1093/nar/gkp464.

\section{Acknowledgements}

We would like to thank the genomics unit at the CNAG for assistance with bulk RNA sequencing. We would like to thank Prof. Gregor Eichele for generously providing us with the Syt10-Cre mice. We thank Veronica Raker for manuscript editing. 


\section{Funding}

Research in the SAB lab is supported partially by the European Research Council (ERC) under the European Union's Horizon 2020 research and innovation programme (Grant agreement No. 787041), the Government of Cataluña (SGR grant), the Government of Spain (MINECO), the La Marató/TV3 Foundation, the Foundation Lilliane Bettencourt, the Spanish Association for Cancer Research (AECC) and The Worldwide Cancer Research Foundation (WCRF). The IRB Barcelona is a Severo Ochoa Center of Excellence (MINECO award SEV-2015-0505). CMG was funded by the European Union's Horizon 2020 research and innovation programme under the Marie Sklodowska-Curie grant agreement 749869. KBK. was supported by an NIH F32 Fellowship - DK121425. PMC acknowledges funding from MICINN-RTI2018-096068， ERC-2016-AdG-741966， LaCaixa-HEALTH-HR1700040, MDA, UPGRADE-H2020-825825, AFM, DPP-Spain, Fundació La MaratóTV380/19-202021, MWRF, and María-de-Maeztu Program for Units of Excellence to UPF (MDM-2014-0370) and the Severo- Ochoa Program for Centers of Excellence to CNIC (SEV-2015-0505). PSW was supported by grant RYC2019-026661-I funded by MCIN/AEI/10.13039/501100011033 and by "ESF Investing in your future". TM received funding from the European Union's Horizon 2020 research and innovation programme under the Marie Skłodowska-Curie grant agreement No. 754510. VMZ received financial support through the "la Caixa" INPhINIT Fellowship Grant for Doctoral Studies at Spanish Research Centers of Excellence, "la Caixa" Foundation, Barcelona (ID 100010434). The fellowship code is LCF/BQ/IN17/11620018. The project has received funding from the European Union's Horizon 2020 research and innovation program under the Marie Skłodowska-Curie grant agreement no. 713673 .

\section{Author contributions}

TM, VMZ, PSW and SAB designed the study with critical input from all other authors. TM and VMZ performed the majority of experiments. EG provided experimental assistance with the analysis of the cell cycle by FACS. PP performed western blot and immunofluorescence experiments characterising Bmal1 expression. TM, VMZ and CL performed the data analysis of the diurnal and circadian transcriptomes. PSW and SF generated the Bmal-StopFL mice 
used in this study. TM and SAB wrote the manuscript, integrating feedback from all other authors.

\section{Competing interests}

SAB is a co-founder and scientific advisor of ONA Therapeutics. The other authors declare no competing interests.

\section{Data and materials availability}

The datasets associated with this study have been uploaded to the Gene Expression Omnibus under the code GSE190035.

\section{Materials and Methods}

\section{Housing of mice and study design}

All mice were bred and maintained in the animal facilities of the Barcelona Science Park (PCB), following the applicable Spanish and European Union regulations. The experimental protocols applied in this paper were approved by the Catalan Government, in line with the relevant legislation and the guidelines of the Institutional Animal Care and Use Committee (IACUC) at the PCB.

The ARRIVE guidelines for reporting and performing animal experiments (39) were taken into consideration during the design of all experiments. In all instances, mice were maintained in rooms at $22^{\circ} \mathrm{C}$ degrees with ad libitum access to standard chow diet. To profile daily transcriptional rhythms, male mice were maintained under standard $12 \mathrm{~h}: 12 \mathrm{~h} \mathrm{light/dark}$ conditions (LD), and four eight-week-old mice were collected per time point. To profile circadian transcription, seven-week-old female mice were maintained in constant darkness (DD) for between 156 and 176 hours and then sacrificed (at eight weeks of age); four mice were harvested per time point. Eight-week-old mice were used to ensure that the mice were within telogen phase when the epidermis was collected; any mouse in anagen phase was excluded. 


\section{Generation of RE/RE mice}

To generate RE/RE mice, Bmal1-stopFL mice (5) were crossed with K14-Cre mice (40) and

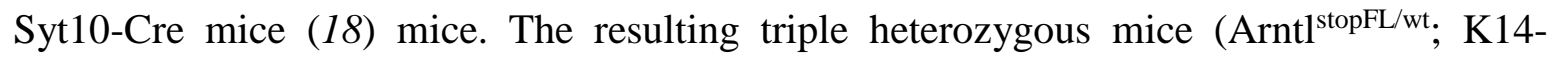
$\left.\mathrm{Cre}^{\mathrm{tg} / \mathrm{wt}} ; \mathrm{Syt} 10-\mathrm{Cre}^{\mathrm{tg} / \mathrm{wt}}\right)$ were then crossed with heterozygous Bmal-stopFL mice $\left(\mathrm{Arntl}^{\mathrm{stopFL} / \mathrm{wt}} ; \mathrm{K} 14-\mathrm{Cre}^{\mathrm{wt} / \mathrm{wt}} ; \mathrm{Syt} 10-\mathrm{Cre}^{\mathrm{wt} / \mathrm{wt}}\right.$ ) to generate the five genotypes used in this manuscript: i) wild-type (WT), Arntt ${ }^{\mathrm{wt} / \mathrm{wt}} ; \mathrm{K} 14-\mathrm{Cre}^{\mathrm{tg} / \mathrm{wt}} ; \mathrm{Syt} 10-\mathrm{Cre}^{\mathrm{tg} / \mathrm{wt}}$; ii) knockout (KO),

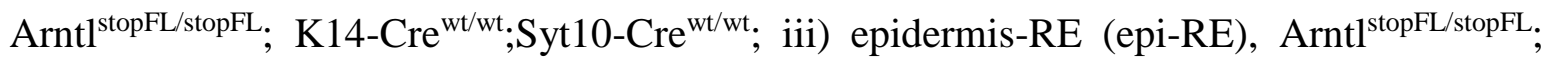
K14-Cre ${ }^{\mathrm{tg} / \mathrm{wt}} ; \mathrm{Syt} 10-\mathrm{Cre}^{\mathrm{wt} / \mathrm{wt}} ;$ iv) brain-RE: Arntl ${ }^{\text {stopFL/stopFL}} ; \mathrm{K} 14-\mathrm{Cre}^{\mathrm{wt} / \mathrm{wt}} ; \mathrm{Syt10-Cre}{ }^{\mathrm{tg} / \mathrm{wt}}$; and v) double reconstituted (RE/RE): Arntl ${ }^{\text {stopFL/stopFL; K14-Cre }}{ }^{\mathrm{tg} / \mathrm{wt}} ; \mathrm{Syt10}-\mathrm{Cre}^{\mathrm{tg} / \mathrm{wt}}$. All initial and maintenance crosses were performed using mice heterozygous for Bmal1-StopFL and each Cre, to avoid sterility associated with total Bmal1 loss or unwanted transgene integration effects, respectively. Only female mice expressing the Syt10-Cre were used for breeding due to the known expression of this Cre in the testes of male mice that may lead to general recombination of the floxed loci (18).

\section{Epidermis extraction}

Mice were sacrificed by $\mathrm{CO}_{2}$ asphyxiation, and death was confirmed by cervical dislocation. The mice were then shaved, and the back skin was subsequently removed and placed in ice cold PBS. A tail sample was also taken to allow the genotype to be revalidated. To remove the hypodermis, the underside of the skin was then vigorously scraped using a scalpel. In a petri dish, the skin was then floated on $10 \mathrm{ml}$ of $1 \mathrm{mg} / \mathrm{ml}$ dispase (Sigma Aldrich) in PBS for $45 \mathrm{~min}$ at $37^{\circ} \mathrm{C}$, to enzymatically separate the epidermis. Subsequently, using a scalpel, the epidermis was scraped from the surface of the skin and then mechanically disaggregated using surgical scissors. The disaggregated epidermis was then placed into $10 \mathrm{ml}$ of ice cold PBS $+10 \%$ chelated FBS to inactivate the dispase. After vigorous shaking, the epidermal extract was sequentially filtered through $100 \mu \mathrm{M}$ and $40 \mu \mathrm{M}$ filters to give a purified extract of epidermal cells. Cell viability was checked via Trypan blue staining, and pellets of one million cells were then lysed in $500 \mu \mathrm{l}$ of TRIzol (Invitrogen) for subsequent RNA extraction. 


\section{Cell cycle analysis by flow cytometry}

Epidermal cells were first extracted from 8-week-old male mice as described in 'Epidermis extraction' and then fixed in ice-cold $70 \% \mathrm{EtOH}$ at $-20^{\circ} \mathrm{C}$ for 2 hours. The cells were then washed $2 \times$ with PBS $+2 \%$ FBS $+1 \mathrm{mM}$ EDTA (wash buffer). The fixed cells were resuspended to $1 \times 10^{7}$ cells $/ \mathrm{ml}$ in wash buffer, and $100 \mu 1$ of cell suspension was then mixed with $20 \mu \mathrm{l}$ FITC anti-Ki67 antibody (BD Biosciences, 556026) and incubated at roomtemperature for $20 \mathrm{~min}$. The cells were then washed $1 \times$ with wash buffer and resuspended in $500 \mu \mathrm{l}$ wash buffer $+5 \mu \mathrm{g} / \mathrm{ml}$ propidium iodide. The proportion of actively cycling cells (Ki67-positive) in G1, S and G2 phase of the cell cycle (cell DNA content: $1 \times-\mathrm{G} 1,>1 \times$ and $<2 \times-\mathrm{S}, 2 \times-\mathrm{G} 2$ ) was interrogated using a using a Gallios flow cytometer (Beckman Coulter) and subsequent data analysis with FlowJo (v.10.8.0). Quantification was performed using a minimum of 10,000 cells/sample.

\section{RNA extraction}

To extract total RNA, epidermal cells lysed in $500 \mu 1$ TRIzol (Invitrogen) were vortexed with $100 \mu \mathrm{l}$ chloroform, centrifuged for $15 \mathrm{~min}$ at $18,000 \times g$ at $4^{\circ} \mathrm{C}$, and then the upper aqueous layer containing RNA was isolated. The aqueous layer was then mixed with $825 \mu 1$ RLT buffer (Qiagen) and $625 \mu 1$ 100\% ethanol to induce RNA precipitation. Samples were then processed using an RNeasy mini kit (Qiagen) according to the manufacturer's protocol, and RNA was eluted in molecular biology-grade water and stored at $-80^{\circ} \mathrm{C}$.

\section{Western blot}

Frozen tissue was added to RIPA buffer (50 mM Tris- $\mathrm{HCl}$ [pH 8.0], 1\% NP-40, 0.1\% SDS, $150 \mathrm{mM} \mathrm{NaCl}, 5 \mathrm{mM} \mathrm{MgCl} 2,0.5 \%$ sodium deoxycholate, and $1 \mathrm{mM}$ PMSF) supplemented with $330 \mathrm{nM}$ trichostatin A (Sigma), Protease Inhibitor Cocktail (Roche), $20 \mathrm{mM} \mathrm{NaF,} 1 \mathrm{mM}$ DTT, and $10 \mathrm{mM}$ nicotinamide and then lysed with $10 \mathrm{sec}$ of sonication at $60 \%$ power using a VWR 200 homogenizer (Avantor). The resulting lysates were centrifuged for 15 min at $13.2 \times 10^{6} \mathrm{~g}$ at $4^{\circ} \mathrm{C}$, and the supernatant was retained. Protein concentrations were quantified by Bradford assay (Bio-Rad). Next, 10-20 $\mu \mathrm{g}$ of protein was separated in an $8 \%$ polyacrylamide gel and transferred to nitrocellulose membranes, which were incubated with anti-BMAL1 (Abcam, ab93806) or anti-P84 (Genetex, GTX70220) overnight at $4^{\circ} \mathrm{C}$. The 
membranes were subsequently incubated with peroxidase-conjugated secondary antibodies (anti-mouse IgG, HRP conjugate, EMD Millipore, AP160P; anti-rabbit IgG, HRP-linked, EMD Millipore, 12-348) at room temperature for 1 hour, and then visualized using chemiluminescent HRP substrate (WBKLS0500, EMD Millipore).

\section{Immunofluorescence staining}

Freshly-extracted brains were frozen in isopentane at $-80^{\circ} \mathrm{C}$ and then cut into $10 \mu \mathrm{m}$ sections with a CM1950 Cryostat (Leica). After cutting, sections were sequentially fixed for 20 min in ice cold 4\% PFA, permeabilized with PBS $+0.3 \%$ Triton $\mathrm{X}-100$ for $15 \mathrm{~min}$ at room temperature, and blocked with PBS plus 5\% BSA and 10\% normal goat serum for two hours at room temperature. Sections were then incubated with an anti-BMAL1 antibody (Novus, NB100-2288) and diluted in blocking buffer overnight at $4^{\circ} \mathrm{C}$. Finally, sections were incubated with Alexa Fluor 488 goat anti-Rabbit IgG (Invitrogen, A-11008) for 2 hours at room temperature. Nuclei were stained using DRAQ7 (Biostatus).

\section{RNA sequencing}

Total RNA from mice was quantified by Qubit® RNA BR Assay kit (Thermo Fisher Scientific) and the RNA integrity was estimated by using RNA 6000 Nano Bioanalyzer 2100 Assay (Agilent). RNA-seq libraries were prepared with KAPA Stranded mRNA-Seq Illumina ${ }^{\circledR}$ Platforms Kit (Roche) following the manufacturer's recommendations. Briefly, following mRNA fragmentation, $500 \mathrm{ng}$ of total RNA was used for the poly-A fraction enrichment with oligo-dT magnetic beads. Strand specificity was achieved for second-strand synthesis by using dUTP rather than dTTP. The blunt-ended, double-stranded cDNA was 3'adenylated, and Illumina platform-compatible adaptors with unique dual indexes and unique molecular identifiers (Integrated DNA Technologies) were ligated onto them. The ligation product was enriched over 15 PCR cycles, and the final library was validated on an Agilent 2100 Bioanalyzer with the DNA 7500 assay. Libraries were sequenced using NovaSeq 6000 (Illumina) with a read length of $1 \times 51 b p+17 b p+8 b p$, following the manufacturer's protocol. Image analysis, base calling, and quality scoring of the run were processed using the manufacturer's software Real Time Analysis (RTA 3.4.4). 
Sequencing reads were first subjected to quality control and adapter trimming using Trimmomatic (version 0.36) (41). Reads were then aligned to the mm10 genome (UCSC) using the HISAT2 aligner (version 2.2.1) (42) and subsequently sorted using SAMtools (version 1.3.1) (43). After alignment, reads were quantified using featureCounts (version 1.6.0) (44), and the resulting count data was then normalized to generate RPKM values using the edgeR 'rpkm' function (version 3.18.1) (45).

\section{Identification of circadian genes}

To identify genes exhibiting daily rhythms in expression, the Jonckheere-Terpstra-Kendall (JTK_CYCLE) (46) and BIO_CYCLE (47) algorithms were used. In both instances, RPKM normalised gene expression values ( $\log _{2}$ transformed) were used as input. Genes were considered to be rhythmically expressed if they showed a period of 24 hours and a $P$-value $\leq$ 0.01 .

All heatmaps were generated using the pheatmap package in the $R$ programming environment. The mean of RPKM normalised gene expression values ( $\log _{2}$ transformed) from each time point were used as input and row normalisation was applied to visualise circadian expression patterns. Genes were ordered by their calculated expression phase in the WT condition.

Amplitude and phase plots were generated using values calculated by the JTK_CYCLE algorithm, and all graphs were made using the ggplot2 package in the $\mathrm{R}$ programming environment. To calculate amplitude density, the 'geom_density' function from the ggplot2 package was used to calculate a smoothed density estimate of the amplitude values.

\section{GO, KEGG, and motif analyses}

All gene ontology (GO) and KEGG pathway analyses were performed using the gene list enrichment analysis tool Enrichr (48). GO and KEGG terms found to be enriched within the lists 'GO Biological Processes 2018' and 'KEGG 2019 Mouse', respectively, were analysed further. Enriched terms shown in the figures of this paper were manually filtered to remove redundant terms and to emphasise the enrichment of biologically important categories. The complete output from Enrichr can be found in tables S2 and S3. 
Motif analysis was performed using the online portal PSCAN (49). A region from -450 to +50 relative to the transcription start site of each gene was analysed, and motifs from 'Jaspar 2020_NR' were used as a reference of known motifs.

\section{Statistics}

All information related to replicate inclusion/exclusion criteria, total number of replicates and other relevant statistical information is stated in the figure legends for all graphs.

\section{$\underline{\text { Figure legends }}$}

Fig. 1. (A) Schematic representation of the Bmal1-StopFL mouse (5) (left) and the breeding procedure used to generate the RE/RE model (right). The image of the mouse was created using biorender.com. (B) Western blot of BMAL1 protein levels in different murine tissue types. P84 is shown as a loading control. (C) Immunofluorescence staining of BMAL1 in the suprachiasmatic nucleus. Nuclei were counterstained with Draq7. Scale bar indicates $50 \mu \mathrm{M}$. (D and E) Diurnal expression patterns of core clock genes (D) and clock-controlled genes (E) in the epidermis of all clock reconstitution conditions, as determined by RNA-seq. Values represent the $\log _{2}$-transformed mean ( \pm standard deviation) of four biological replicates. Time point ZT0 is double-plotted for better visualization. BRE/brain-RE, brain-reconstituted (brain-RE); epi-RE, reconstituted in epidermis; KO, knockout of Bmal1-StopFL (wholebody Bmal1 null); RE/RE, double-reconstituted; RPKM, reads per kilobase per million mapped reads; SCN, suprachiasmatic nucleus; Syt10, synaptotagmin 10; WAT, white adipose tissue; WT, wild-type; ZT, Zeitgeber time.

Fig. 2: (A) Quantification of the epidermal diurnal transcriptome in each clock reconstitution condition (left). Venn diagram showing the intersect of the diurnal transcriptomes (right). Rhythmicity was defined as a JTK_CYCLE-calculated periodicity of 24 hours and a $P$ value $<0.01$. (B) Pie chart quantifying the portions of the wild-type (WT) diurnal transcriptome shared with other clock reconstitution conditions. Percentage values are rounded to the nearest whole number. (C) Heat map showing the diurnal expression patterns of genes rhythmic only in the WT epidermis (1589 genes), or only in WT and doublereconstituted (RE/RE) epidermis (651 genes), in all clock reconstitution conditions. Colors represent RPKM values normalized by row. Each condition is internally normalized. Genes are sorted by expression phase. (D and E) Circular histograms (D) and density plots (E) indicating the expression phases and expression amplitudes (respectively), in WT and double-reconstituted epidermis, of genes rhythmically expressed only in epidermis from WT and double-reconstituted mice but not in other clock reconstitution conditions (651 genes). 
(F) Selected KEGG terms enriched amongst genes rhythmically expressed only in WT epidermis ( $n=1589$ genes) or only in WT+RE/RE epidermis (651 genes) (see C). (G) Selected gene ontology terms (cell cycle-related) enriched amongst the diurnal transcriptomes of epidermis from different clock reconstitution conditions. (H) Diurnal expression patterns of late cell cycle regulators in the epidermis of each clock reconstitution condition, as determined by RNA-seq. Values represent the $\log _{2}$-transformed mean $( \pm$ standard deviation) of four biological replicates. The time point ZT0 is double-plotted for better visualization. (I) Percentage of actively cycling epidermal cells (Ki67-positive) in G2 phase $(2 \times$ DNA content) at ZT4 or ZT16 in each clock reconstitution condition. Values represent the mean ( \pm standard deviation) of $n=4$ to 6 biological replicates. $* P<0.05$, ** $P<$ 0.01. (J) Enrichment of transcription factor binding sites in the promoter ( -450 to +50 bases relative to the transcription start site) of genes rhythmic in WT only or in WT+RE/RE only (see C). The calculated $P$-value for each motif in both genesets is shown to emphasize the geneset-specific enrichment. BRE/brain-RE, brain-reconstituted; Epi-RE, reconstituted in epidermis; LD, 12h:12h light/dark cycle; RE/RE, double-reconstituted; RPKM, reads per kilobase per million mapped reads; ns, non-significant; WT, wild-type; ZT, Zeitgeber time.

Fig. 3. (A) Venn diagram indicating the shared-rhythmicity genes (67 genes)-i.e., genes that were rhythmic in the epidermal diurnal transcriptomes of all clock reconstitution conditions. (B) Pie chart indicating the number of the 67 shared-rhythmicity genes (see A) that also exhibited rhythmicity in the epidermis of Bmal1-StopFL mice (whole-body Bmal1 null). Data for Bmal1-StopFL mice were sourced from Welz et al, 2019, and rhythmicity was defined as a JTK_CYCLE calculated periodicity of 24 hours and $P<0.01$. (C) Selected gene ontology terms enriched in the 67 shared-rhythmicity genes (see A). (D) Circular histograms indicating the expression phases of the 67 shared-rhythmicity genes in the epidermis of each clock reconstitution condition. (E) Circular histograms indicating the expression phases of genes rhythmically expressed in both the epidermis of wild-type and brain-reconstituted mice, but not in the shared-rhythmicity gene set (see A), in wild-type and brain-reconstituted epidermis (560 genes). (F) Diurnal expression patterns of early cell cycle regulators and DNA replication machinery in the epidermis of each clock reconstitution condition, as determined by RNA-seq. Values represent the $\log _{2}$-transformed mean ( \pm standard deviation) of $n=4$ biological replicates. The time point ZT0 is double-plotted for better visualization. BRE/brain-RE, brain-reconstituted; RE, reconstituted; RE/RE, double-reconstituted; RPKM, reads per kilobase per million mapped reads; WT, wild-type; ZT, Zeitgeber time.

Fig. 4. (A) Quantification of the epidermal circadian transcriptome in each clock reconstitution condition (left). Venn diagram showing the intersect of the circadian transcriptomes (right). Circadian expression was defined as a JTK_CYCLE-calculated periodicity of 24 hours and $P<0.01$. ( $\mathbf{B}$ and $\mathbf{H}$ ) Diurnal expression patterns of core clock genes $(B)$ and late cell cycle regulators $(\mathrm{H})$ in the epidermis of each clock reconstitution 
condition, as determined by RNA-seq. Values represent the $\log _{2}$-transformed mean $( \pm$ standard deviation) of four biological replicates. The time point CT0 is double-plotted for better visualization. (C) Pie chart quantifying the portions of the wild-type circadian transcriptome shared with other clock reconstitution conditions. Percentage values are rounded to the nearest whole number. (D) Heat map showing the circadian expression changes of genes rhythmic only in the wild-type (WT) epidermis ( $n=1473$ genes) or in epidermis from WT or double-reconstituted (WT + RE/RE) mice ( $n=938$ genes), in all clock reconstitution conditions. Colors represent RPKM values normalized by row. Each condition is internally normalized. Genes are sorted by expression phase. ( $\mathbf{E}$ and $\mathbf{F}$ ) Circular histograms (E) and density plots (F) indicating the expression phases and expression amplitudes (respectively) of the 938 genes rhythmic expressed only in epidermis from WT+RE/RE mice, but not other clock reconstitution conditions (see D). (G) Selected gene ontology terms (cell cycle-related) enriched within the circadian transcriptomes of epidermis from different clock reconstitution conditions. CT, circadian time; DD, dark/dark cycles (e.g., constant darkness); epi-RE, epidermis-reconstituted; RE/RE, double-reconstituted; RPKM, reads per kilobase per million mapped reads; WT, wild-type.

Supplementary Fig. S1. Immunofluorescence staining of BMAL1 in the thalamus and frontal cortex. Nuclei were counterstained with Draq7. Scale bar indicates $50 \mu \mathrm{M}$. BRE/brain-RE, brain-reconstituted; KO, Bmal1 knock-out (Bmal1-StopFL).

Supplementary Fig. S2. (A and B) Circular histograms (A) and density plots (B) indicating the distribution of expression phases and expression amplitudes (respectively) of genes constituting the epidermal diurnal transcriptome of each clock reconstitution condition. (C) Selected gene ontology terms enriched for genes that were rhythmic only in the wild-type (WT) epidermis ( $n=1589$ genes) or in epidermis from WT or double-reconstituted (RE/RE) mice ( $n=651$ genes). (D) Enrichment of selected gene ontology terms in the whole epidermal diurnal transcriptome in each clock reconstitution condition. (E) Diurnal expression patterns of differentiation regulators in the epidermis of each clock reconstitution condition, as determined by RNA-seq. Values represent the $\log _{2}$-transformed mean ( \pm standard deviation) of four biological replicates. Time point ZT0 is double-plotted for better visualization. GO, gene ontology; RE/RE, double-reconstituted; RE, reconstituted.

Supplementary Fig. S3. (A and C) Quantification of the epidermal diurnal (A) and circadian (C) transcriptomes in each clock reconstitution condition using the BIO_CYCLE algorithm (left). Venn diagram showing the intersect of the circadian gene sets (right). Rhythmicity was defined as a BIO_CYCLE-calculated periodicity of 24 hours and a $P$-value $<0.01$. (B and D) Pie charts quantifying the proportion of the wild-type (WT) diurnal (B) and circadian (D) transcriptomes (as defined by BIO_CYCLE) shared with other clock reconstitution conditions. Percentage values are rounded to the nearest whole number. (E and $\mathbf{F}$ ) Venn 
diagrams quantifying the intersect of diurnal $(\mathrm{E})$ and circadian $(\mathrm{F})$ transcriptomes identified using the JTK_CYCLE or BIO_CYCLE algorithms in WT (left) and double-reconstituted (right) epidermis. BRE/brain-RE, brain-reconstituted; DD, dark/dark cycles (e.g., constant darkness); Epi-RE, epidermis-reconstituted; LD, 12h:12h light/dark cycles; RE/RE, doublereconstituted; WT, wild-type.

Supplementary Fig. S4. (A and B) Circular histograms (A) and density plots (B) indicating the distribution of expression phases and expression amplitudes (respectively) of genes constituting the epidermal circadian transcriptome of each clock reconstitution condition. (C) Circadian expression patterns of clock-controlled genes in the epidermis of each clock reconstitution condition, as determined by RNA-seq. Values represent the $\log _{2}$-transformed mean ( \pm standard deviation) of four biological replicates. The time point CT0 is doubleplotted for better visualization. (D) Selected gene ontology terms enriched amongst genes commonly circadian in wild-type, brain-reconstituted, and double-reconstituted epidermis ( $n=109$ genes; genes rhythmic in epidermis-reconstituted epidermis were excluded). (E) Circular histograms indicating the expression phases of the gene set described in (D) in the epidermis of wild-type, brain-reconstituted, and double-reconstituted mice. CT, circadian time; RE/RE, double-reconstituted; RE, reconstituted; RPKM, reads per kilobase per million mapped reads. 


\section{Figure 1}

A

bioRxiv preprint doi: https://doi.org/10.1101/2022.01.26.477844; this version posted January 28, 2022. The copyright holder for this preprint

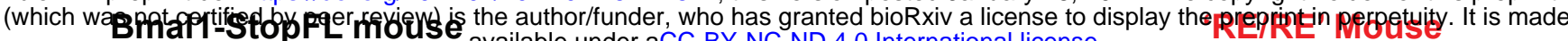

(Welz et al. 2019)

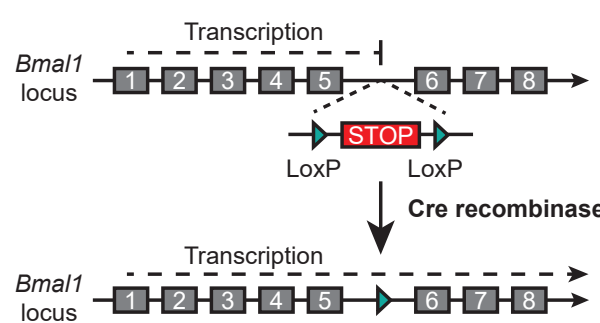

Bmal1 is expressed only in cells expressing Cre-recombinase

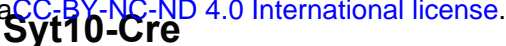

\section{Brain/SCN-specifc}

'Brain-RE' (BRE)

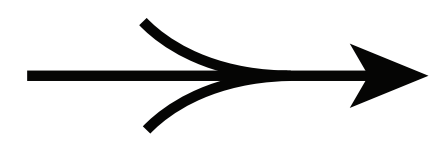

Keratin 14-Cre

Keratinocyte-specific

‘Epidermis-RE’ (Epi-RE)

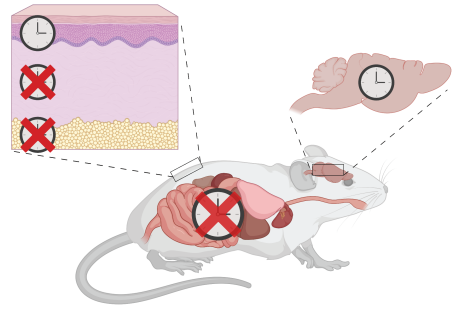

Clock communication restored between only the interfollicular epidermis and the brain
B
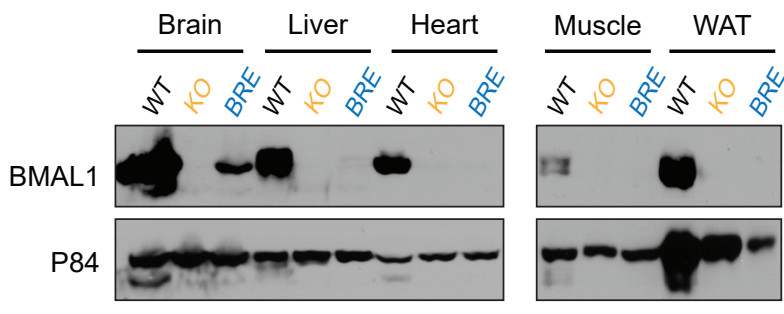

C

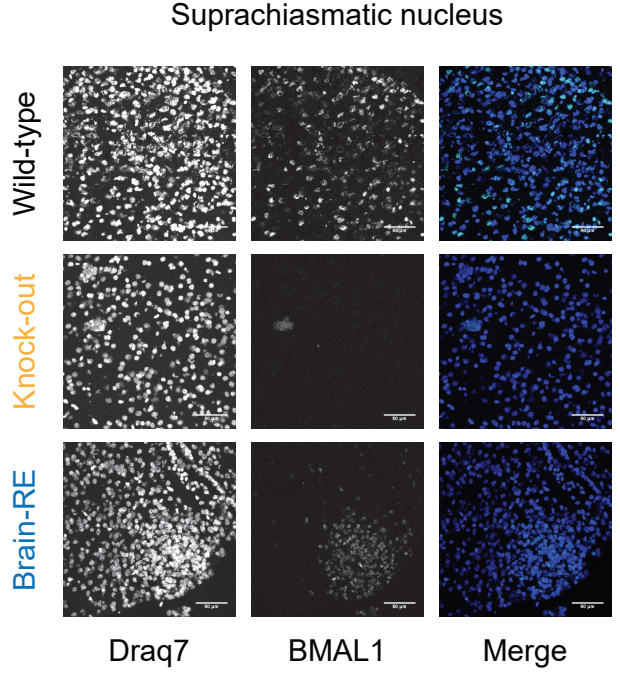

D

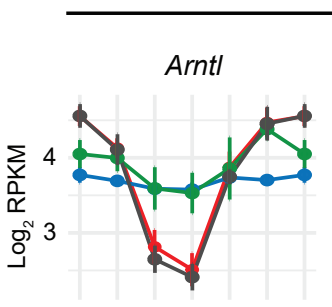

$\begin{array}{lllllll}0 & 4 & 8 & 12 & 16 & 20 & 24\end{array}$

Per1

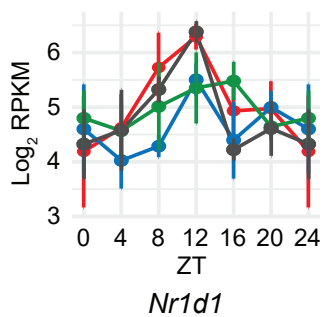

Nr1d1

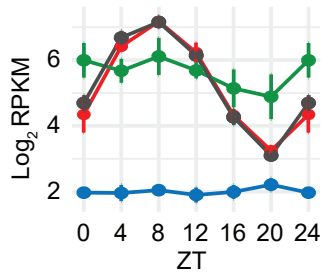

Interfollicular epidermis

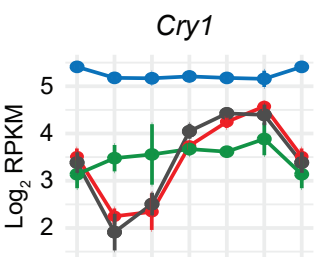

$\begin{array}{lllllll}0 & 4 & 8 & 12 & 16 & 20 & 24\end{array}$

Per2

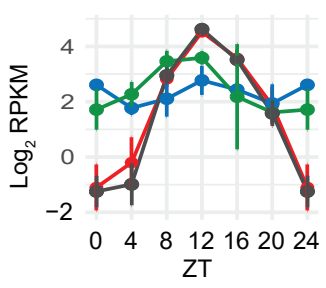

Nr1d2
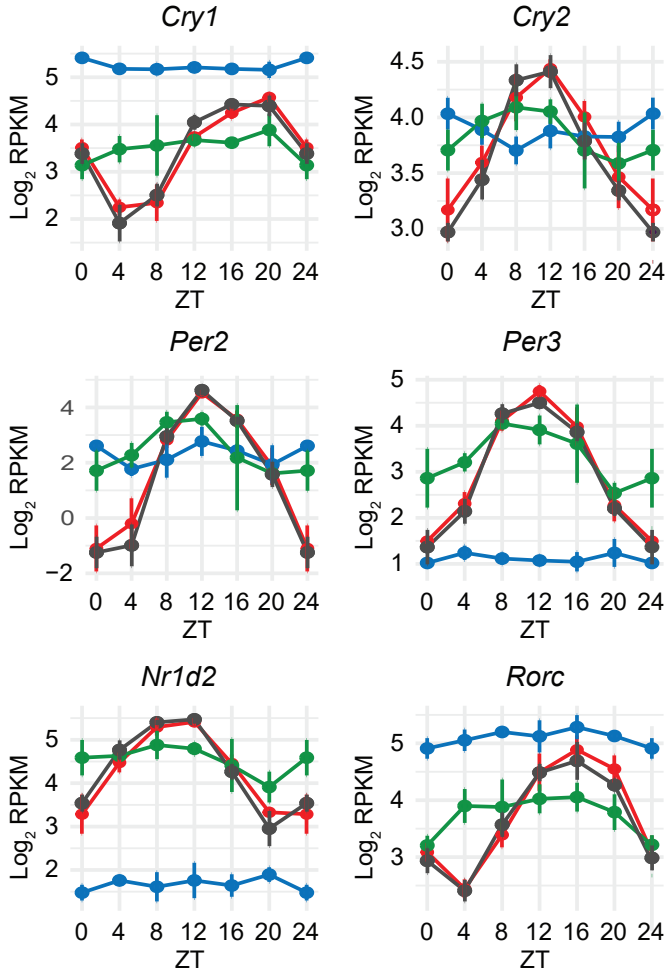

$\begin{array}{lllll}0 & 4 & 8 & 12162024\end{array}$

Per3

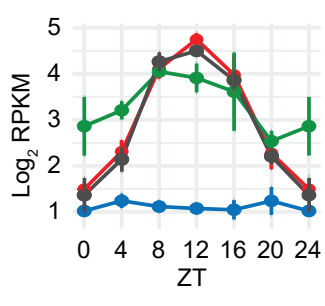

Rorc

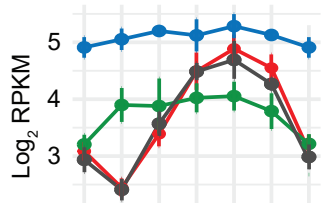

$\begin{array}{lllllll}0 & 4 & 8 & 12 & 16 & 20 & 24\end{array}$ ZT

E
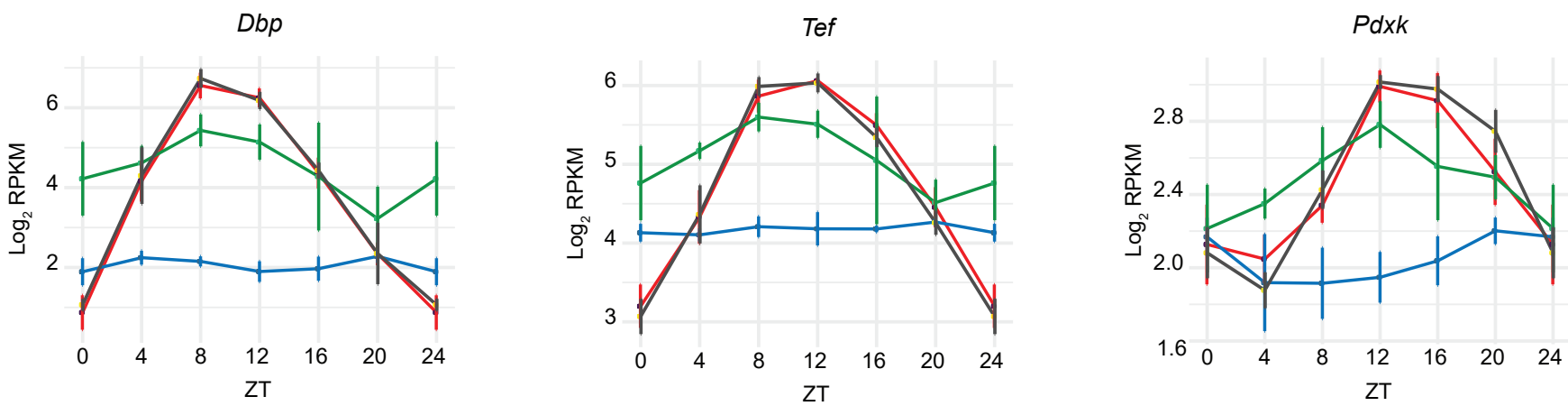

Wild-type $\square$ RE/RE $\square$ Brain-RE $\square$ Epi-RE 


\section{Figure 2}

A

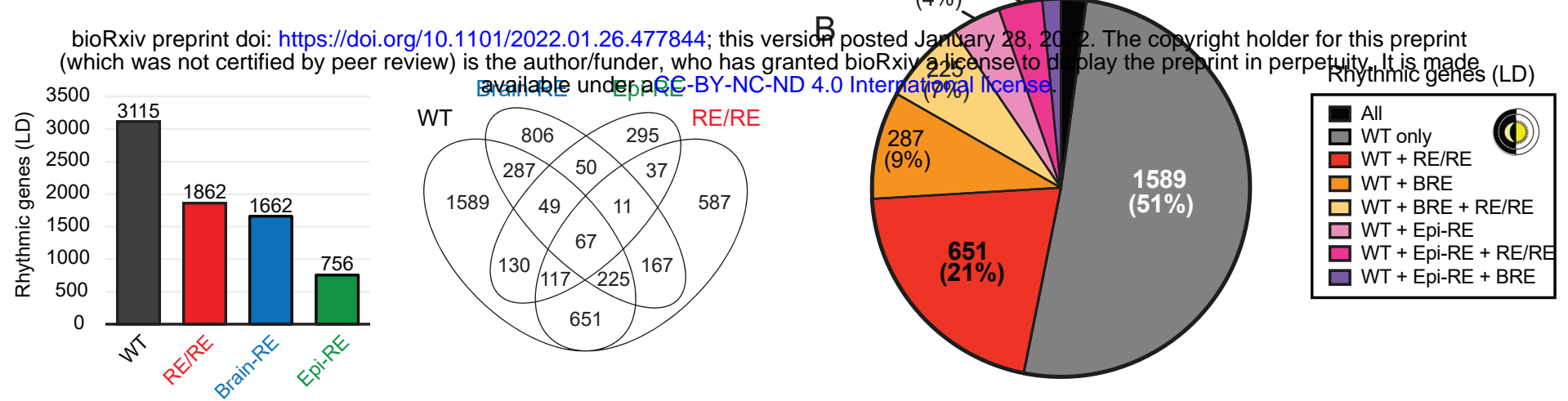

C

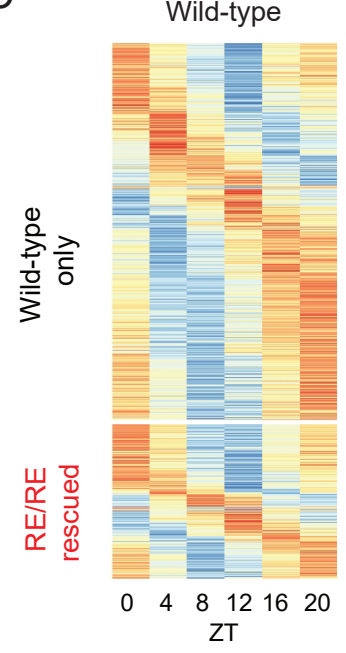

$\mathrm{RE} / \mathrm{RE}$

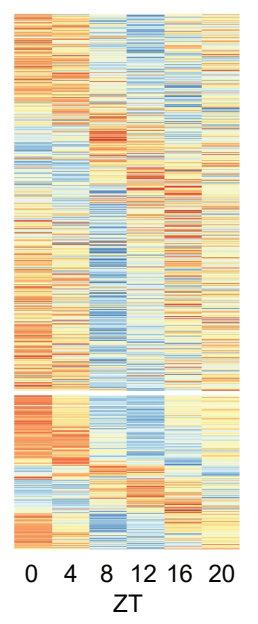

Brain-RE

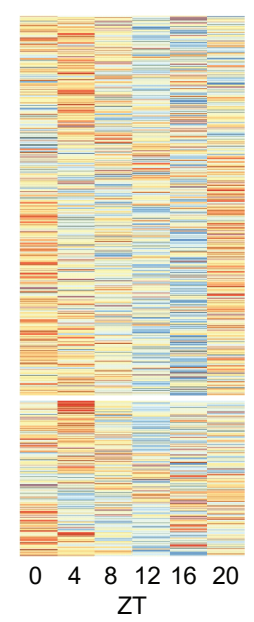

RE/RE-rescued

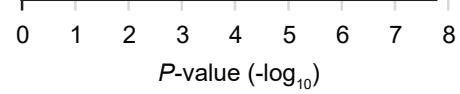

Wild-type only

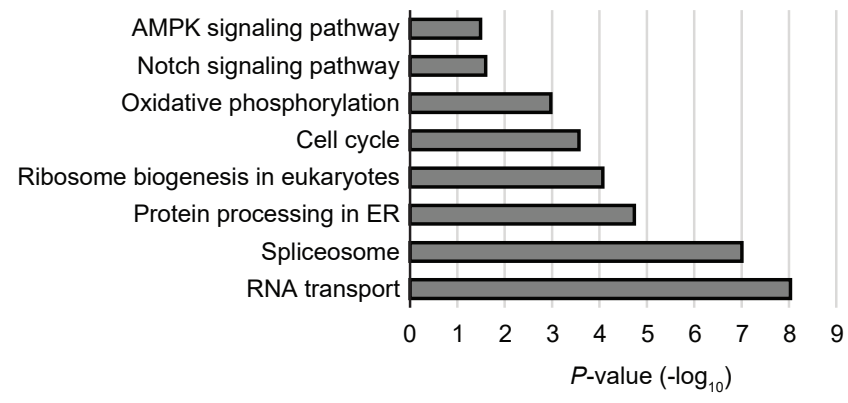

Epi-RE

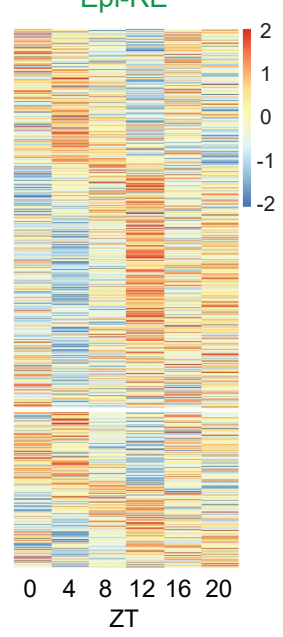

$117 \stackrel{49}{69} \quad 67$

$\begin{array}{ccc}117 & (2 \%) & 67 \\ (2 \%) & \end{array}$

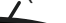

Gap junction $\square$ Citrate cycle (TCA cycle) Protein processing in ER Propanoate metabolism $\mathrm{Ca}^{2+}$ reabsorption Circadian rhythm Cell cycle

F

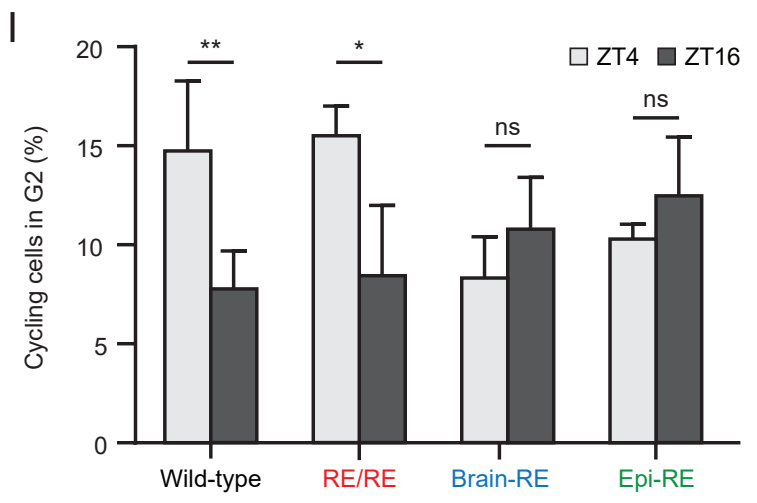

G

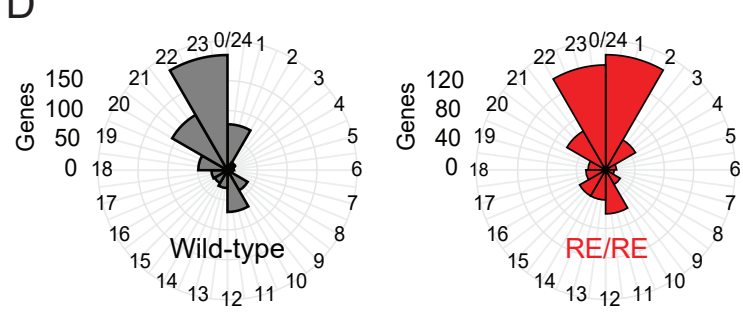

E

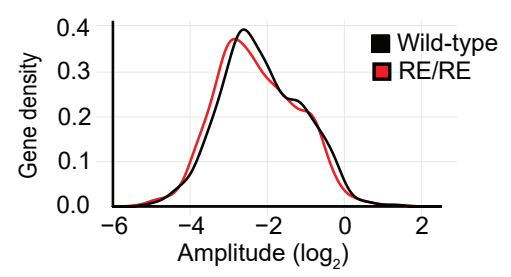

$\mathrm{H}$

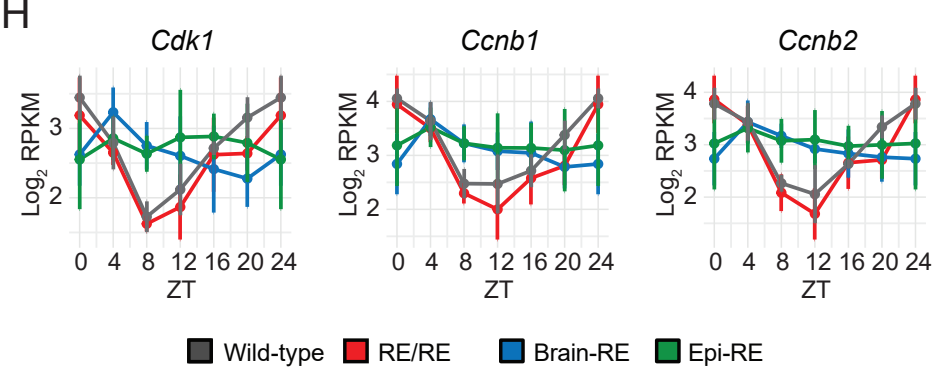




\section{Figure 3}

A

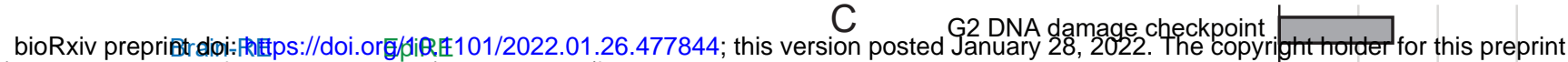
(which was not certified by peer review) is the author/funder, who has granted bioRxiv a license to disptatheprep imt in perpejuity. It is made

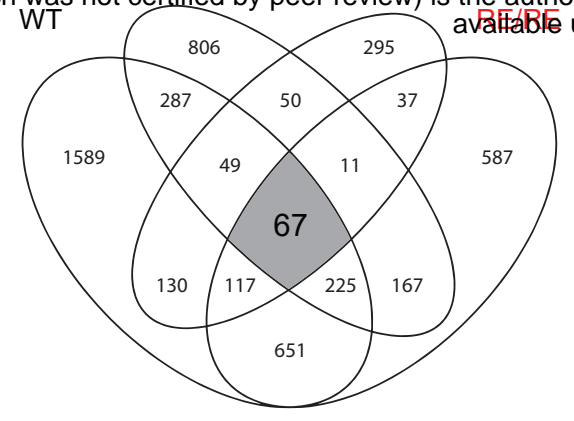

B

$\mathrm{D}$

Cellular response to DNA damage stimulus

DNA replication checkpoint

G1/S transition of mitotic cell cycle

DNA replication

DNA metabolic process
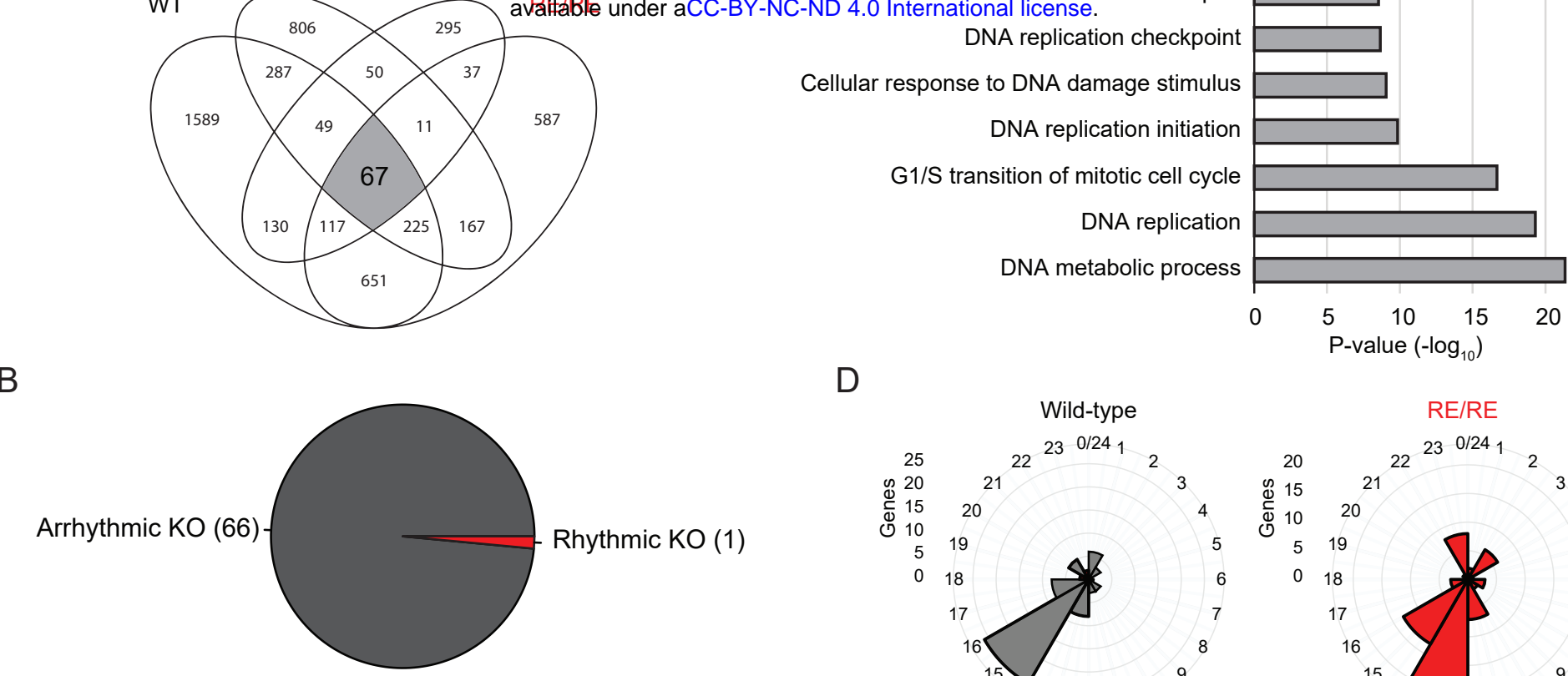

E

Genes rhythmic in WT and Brain-RE - genes common to all conditions excluded

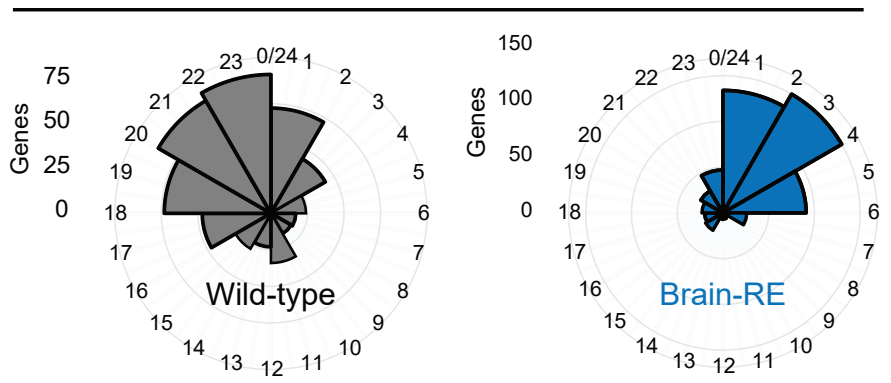

F
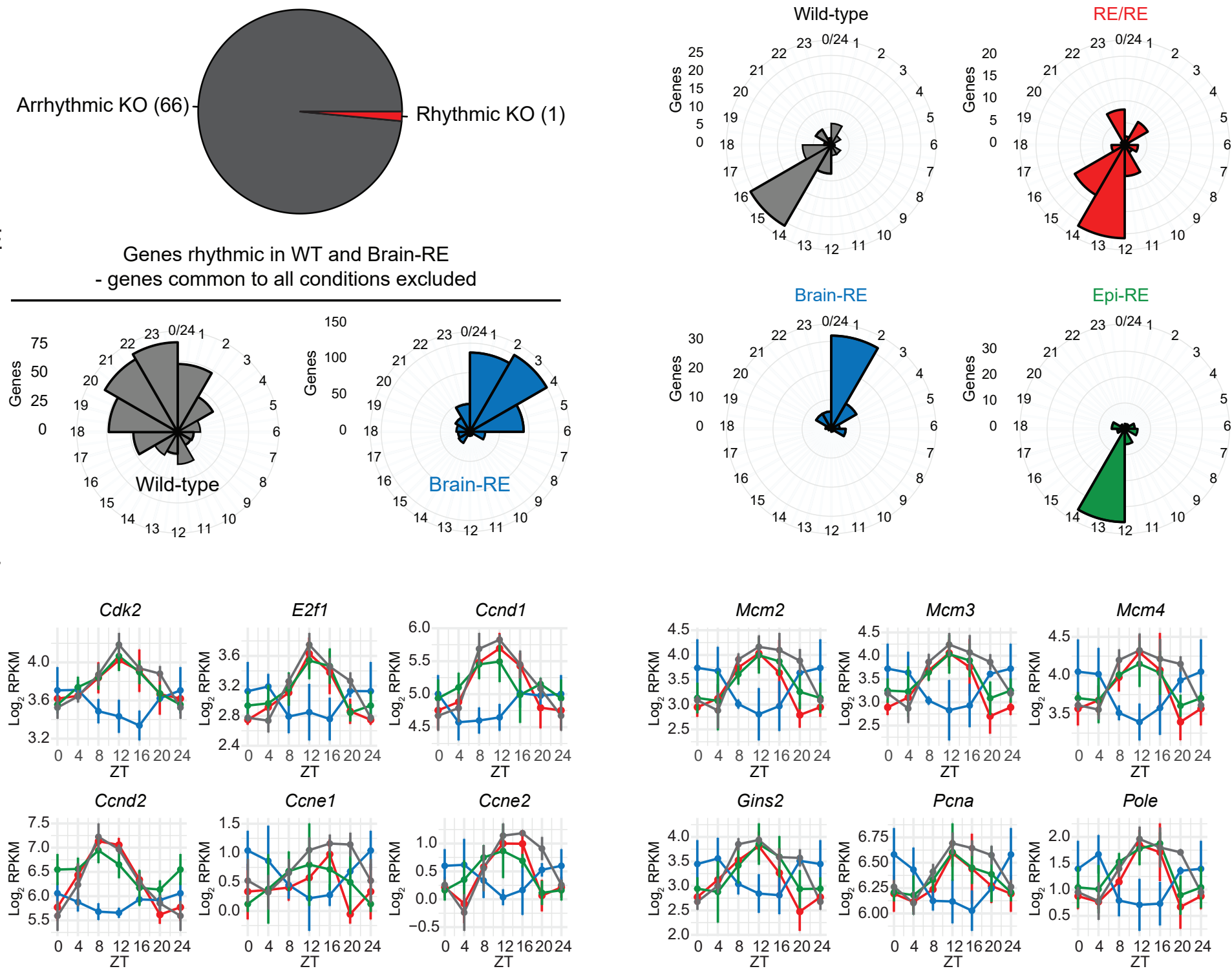

Wild-type $\square$ RE/RE

Brain-RE $\square$ Epi-RE 


\section{Figure 4}

A bioRxiv preprint doi: https://doi.org/10.1101/2022.01.26.477844; this version posted January 28, 2022. The copyright holder for this preprint (which was not certified by peer review) is the auther/funder; who has granted bioRxiv a license to display the preprint in perpetuity. It is made

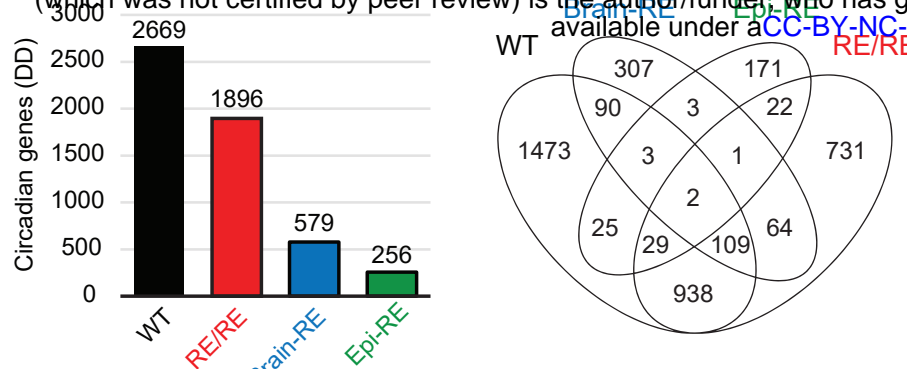

C $\quad 109 \stackrel{25}{25} \quad 29$

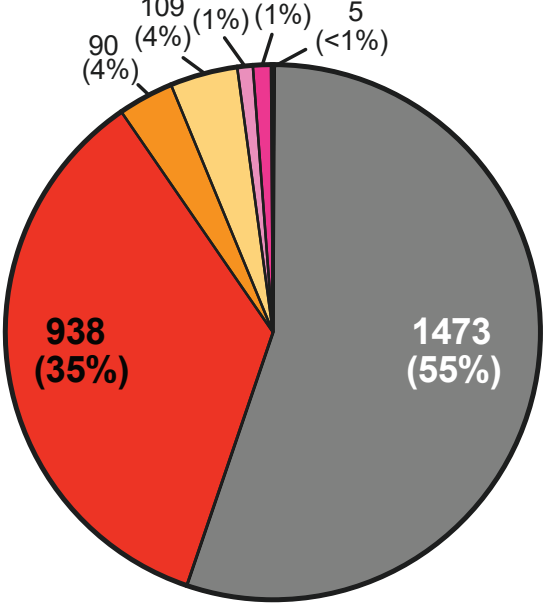

Circadian genes (DD)
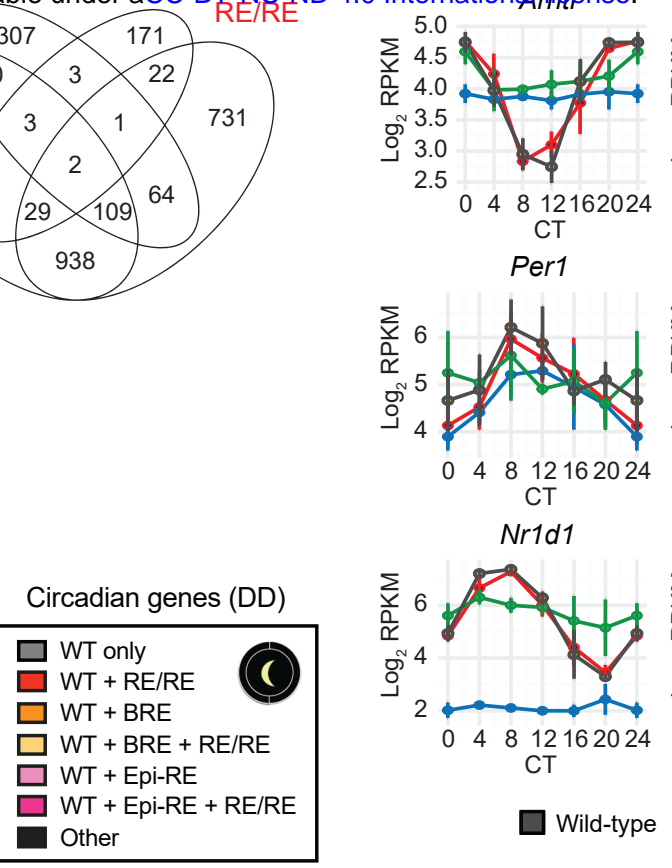

Cry1

Cry2
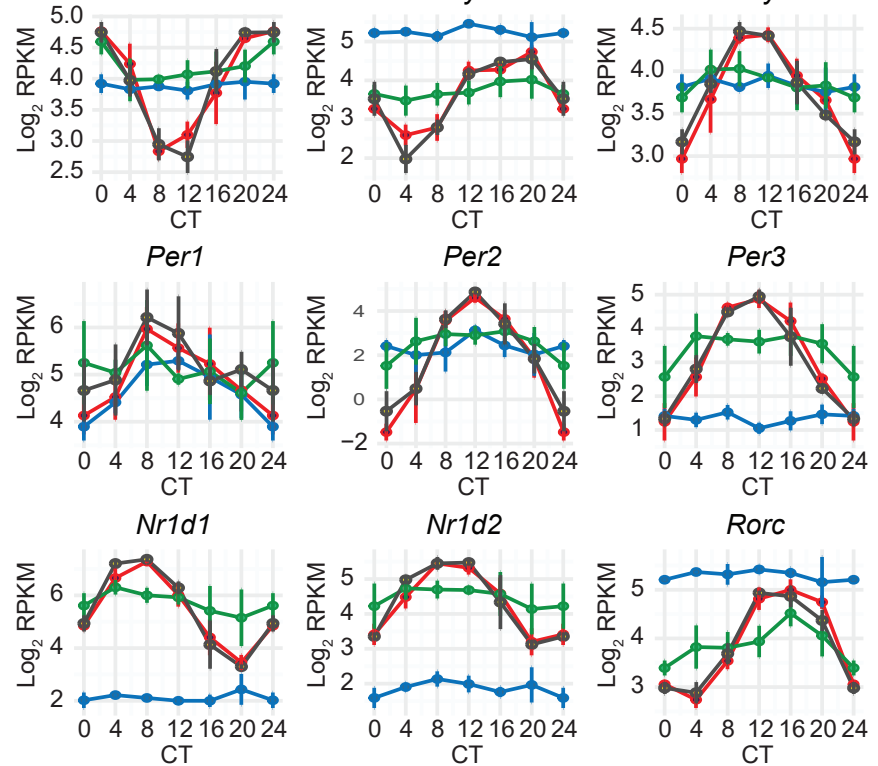

$\square$ Wild-type $\square$ RE/RE $\square$ Brain-RE $\square$ Epi-RE

E

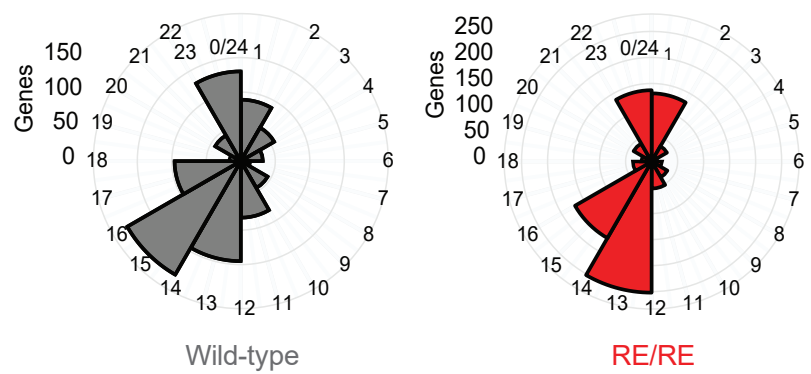

$\mathrm{F}$

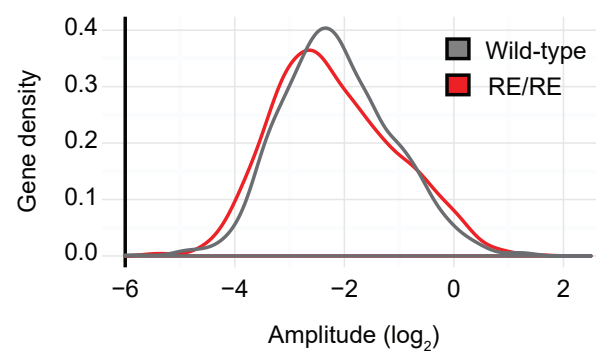

G

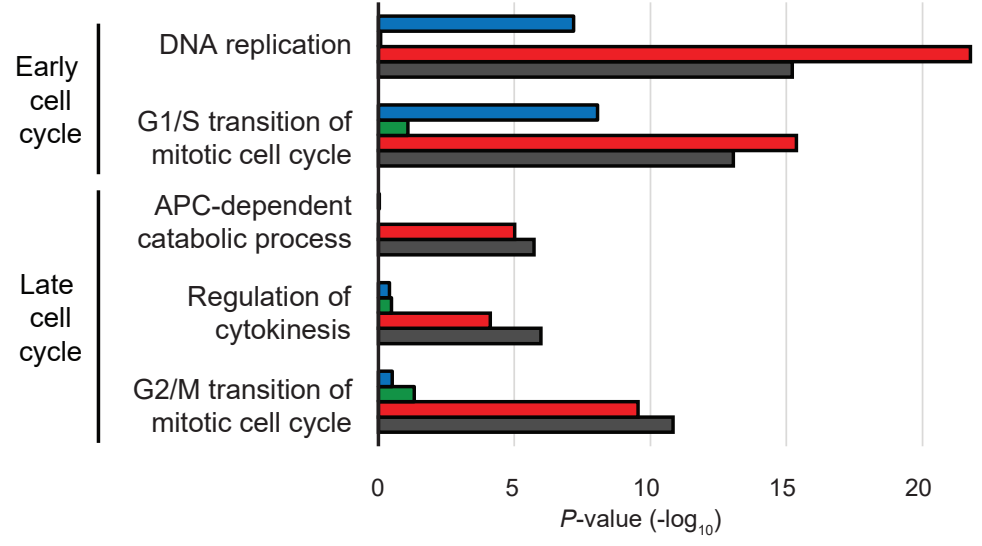

$\mathrm{H}$
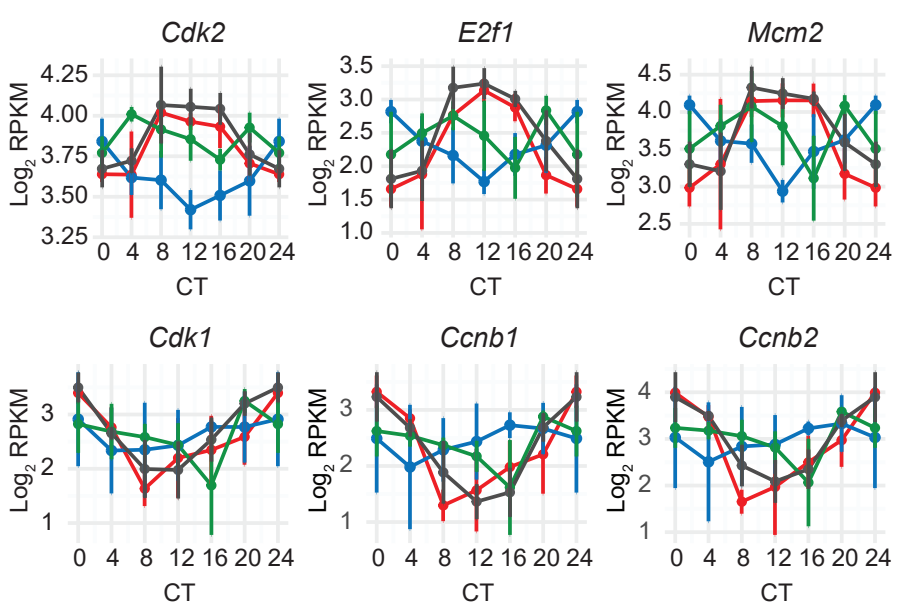


\section{Supplementary Figure 1}

A bioRxiv preprint doi: https://dol.org/10.1101/2022.01.26.477844; this version posted January 28, 2022 . The copyright holder for this preprint

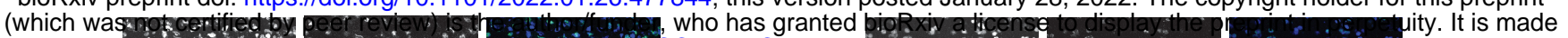

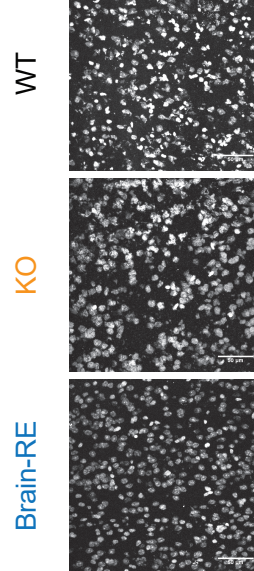

DRAQ7
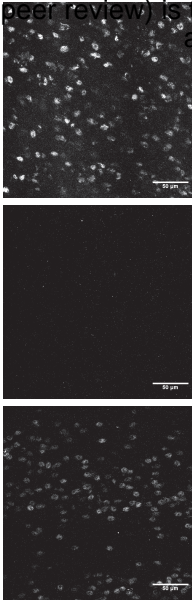

BMAL1
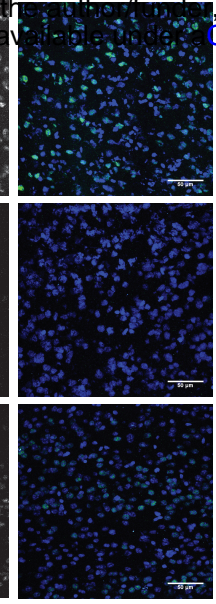

Merge

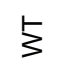

而
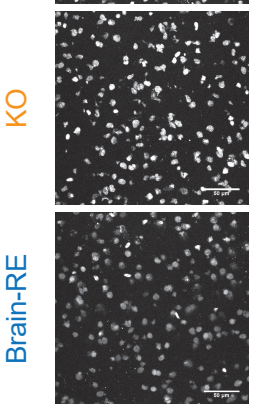

DRAQ7

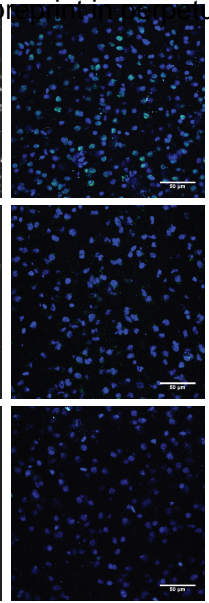

Merge

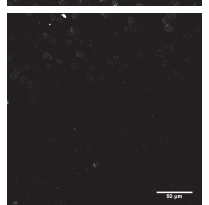

BMAL1

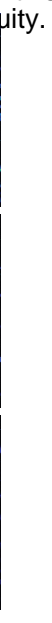
ity. It is made 


\section{Supplementary Figure 2}

A bioRxiv preprint doi: https://doi.org/10.1101/2022.01.26.477844; this version posted January 28, 2022. The copyright holder for this preprint

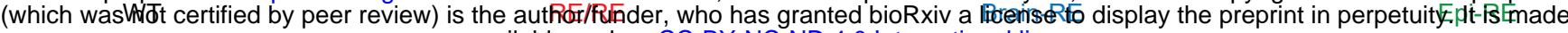
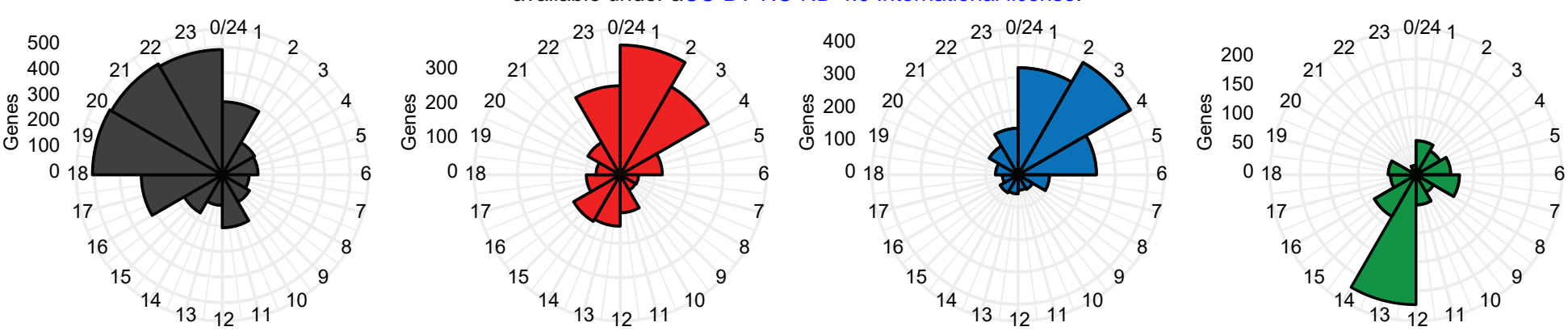

B

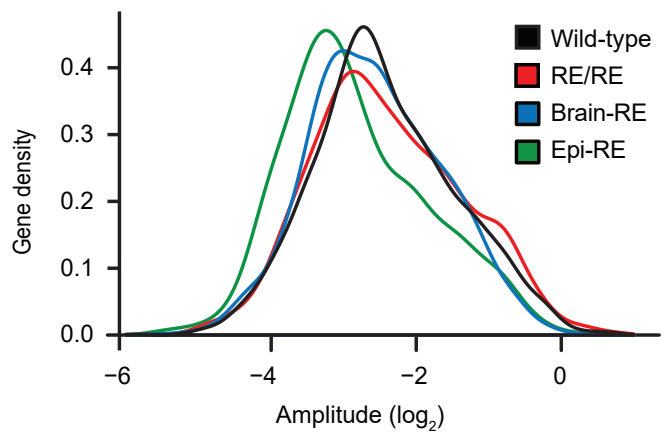

C

GO terms - Wild-type only
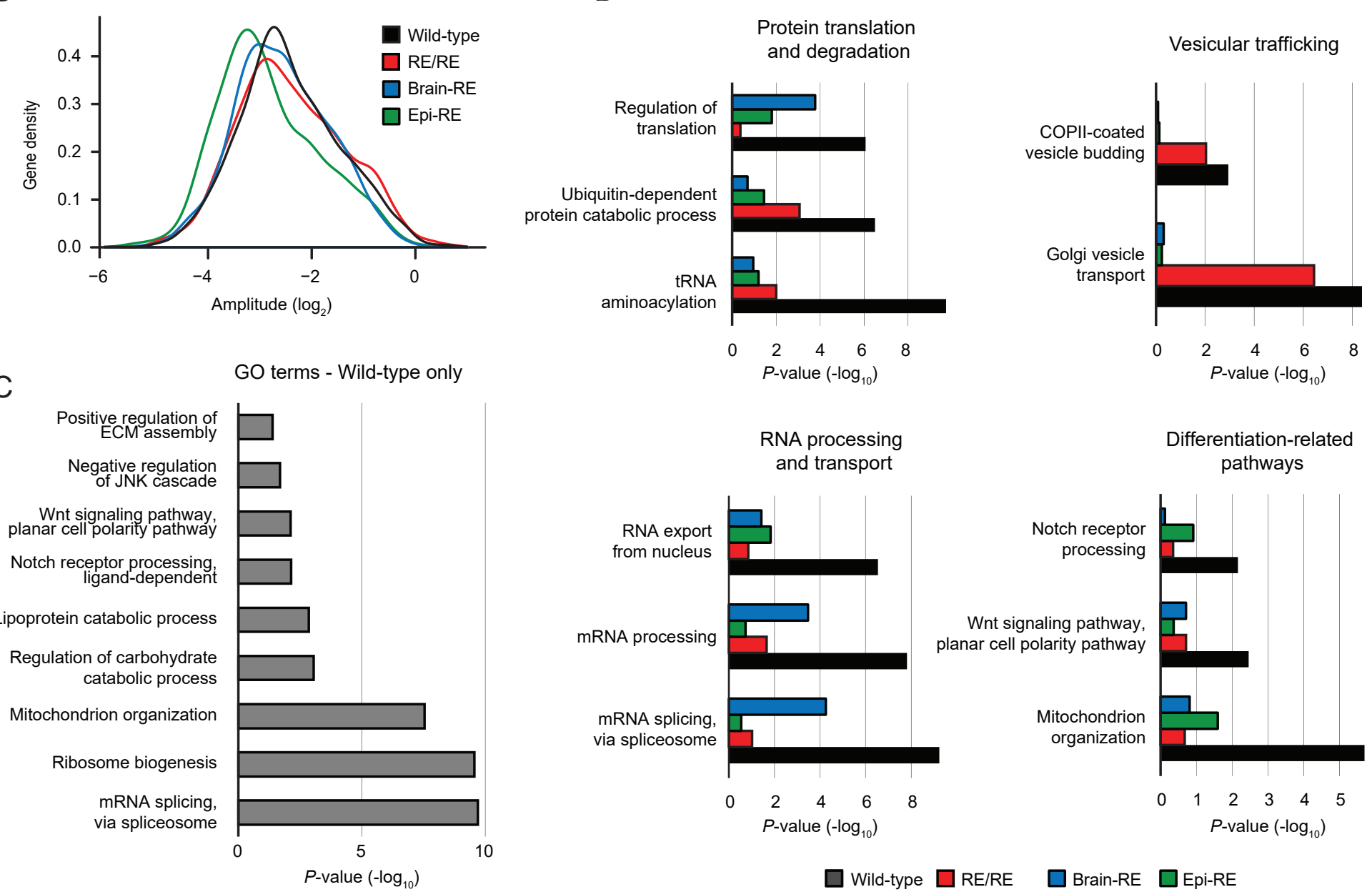

GO terms - RE/RE rescued

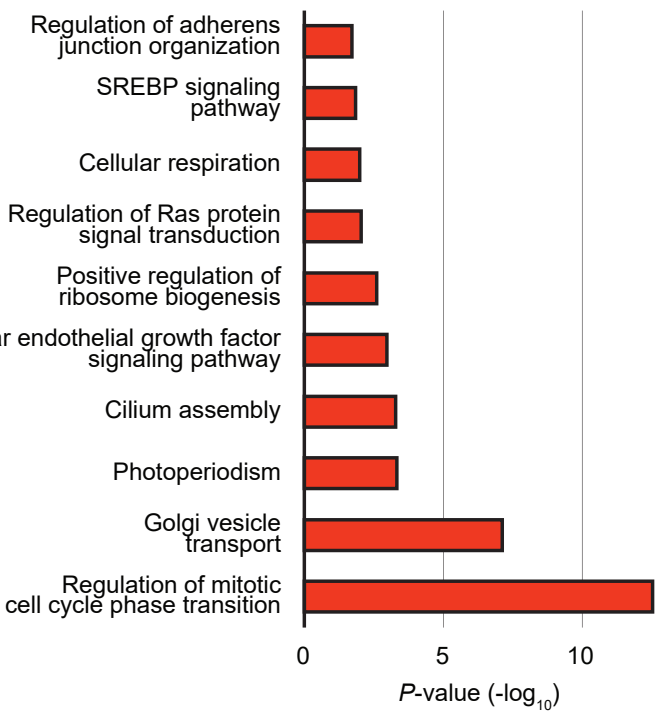

$E$

DII1

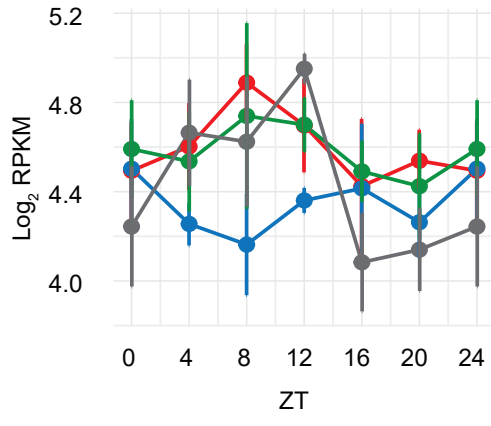

Fzd2

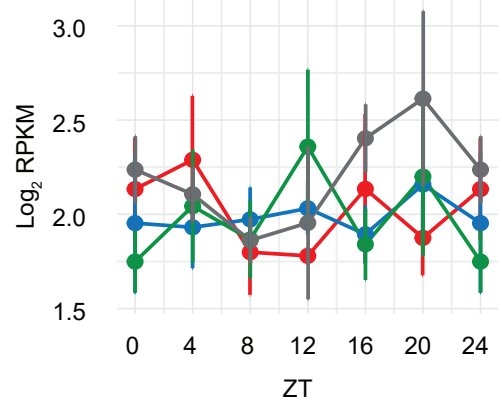

Wild-type $\square$ RE/RE $\square$ Brain-RE $\square$ Epi-RE 


\section{Supplementary Figure 3}

A bioRxiv preprint doi: https://doi.org/10.1101/2022.01.26.477844; this version posteßJanuary 28, 2022. The copyright holder for this preprint 250 (which was not certified by peer review) is the author/funder, who has granted bioRxiv a license to display the preprint in perpetuity. It is made

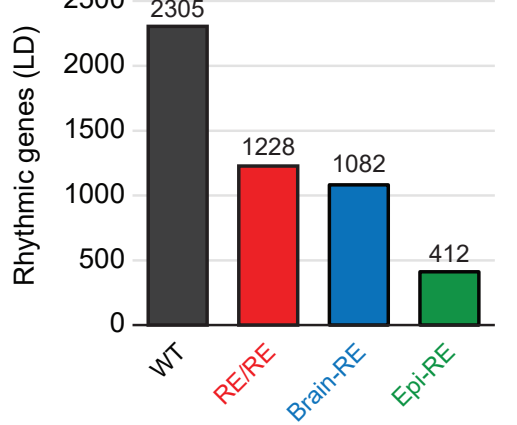

C

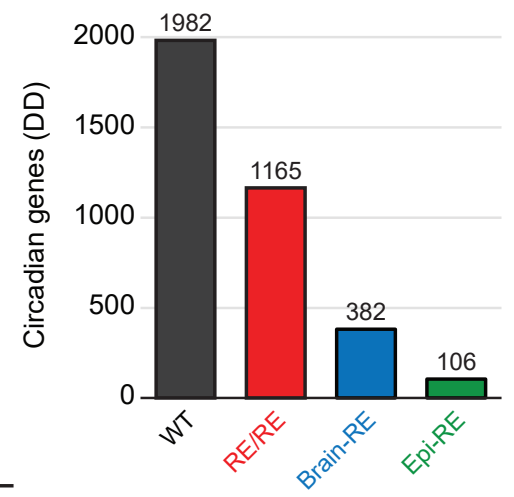

$E$

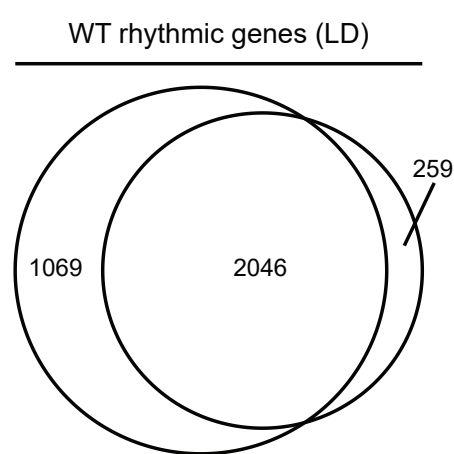

JTK_CYCLE BIO_CYCLE
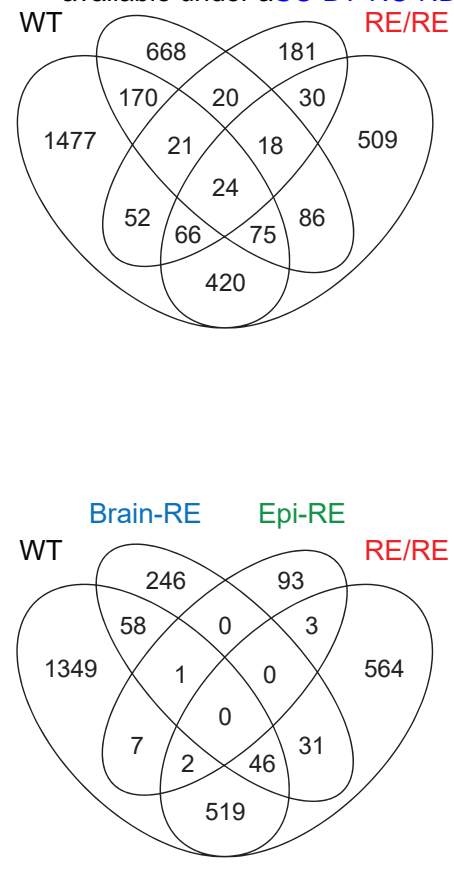

F

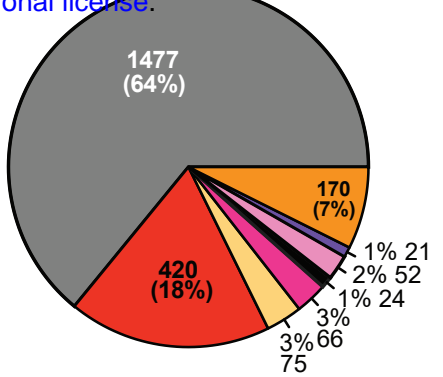

Rhythmic genes (LD)

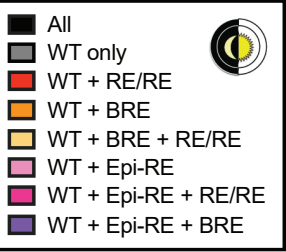

D
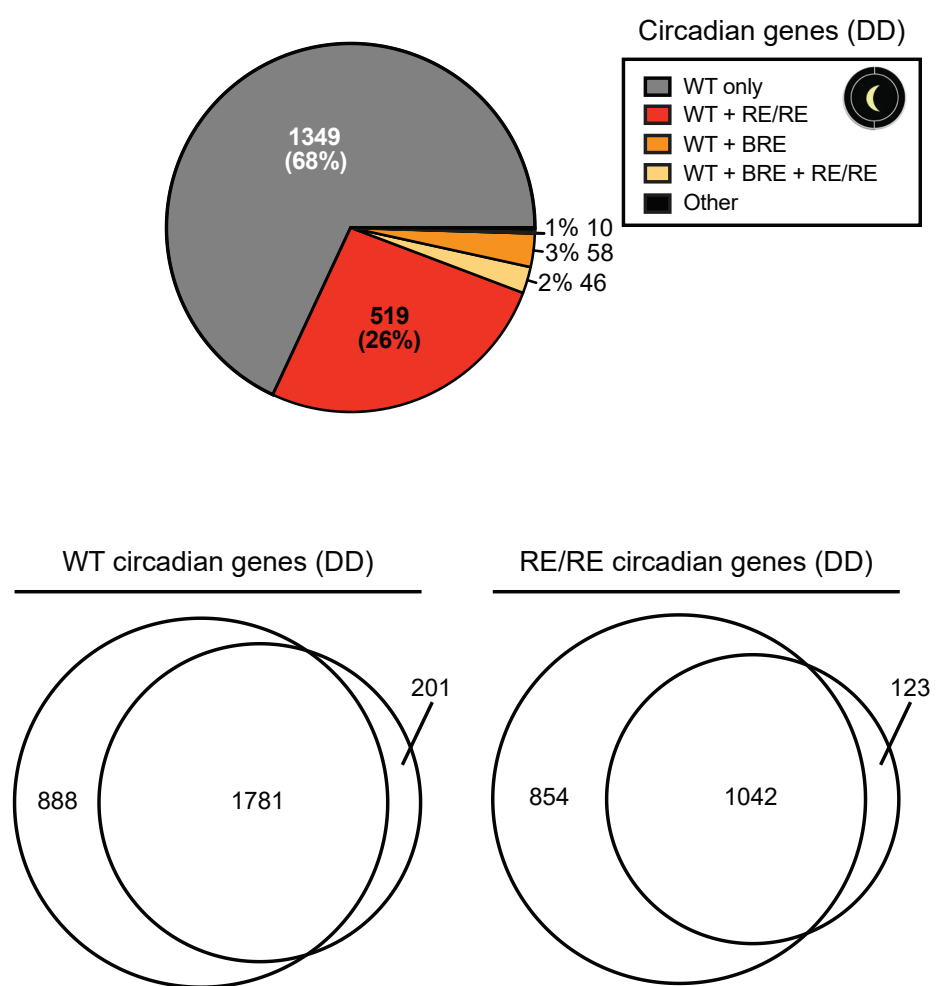

JTK CYCLE BIO_CYCLE
JTK CYCLE BIO CYCLE 


\section{Supplementary Figure 4}

bioRxiv preprint doi: https://doi.org/10.1101/2022.01.26.477844; this version posted January $28,2022$. The copyright holder for this preprint
A (which was not certified by peer review) is the author/funder, who has gFanted bioRxiv a license to display the preprint in perpetuity. It is made Wild-type

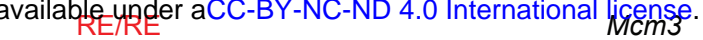

Mcm4

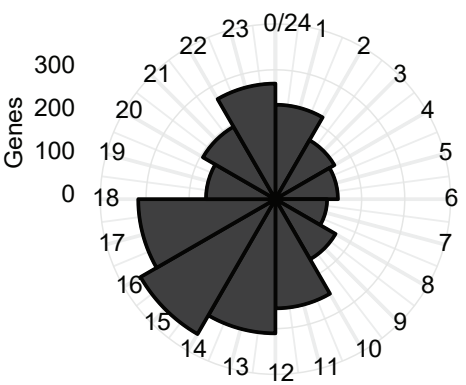

Brain-RE

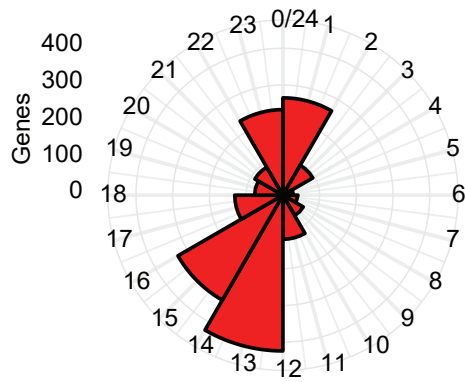

Epi-RE
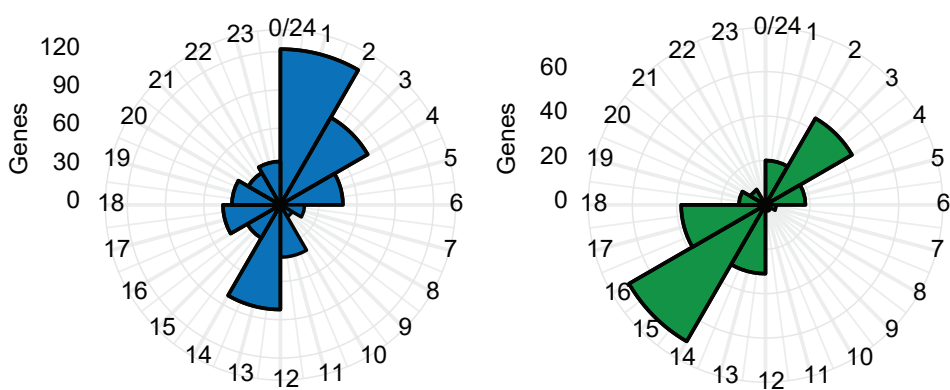

B

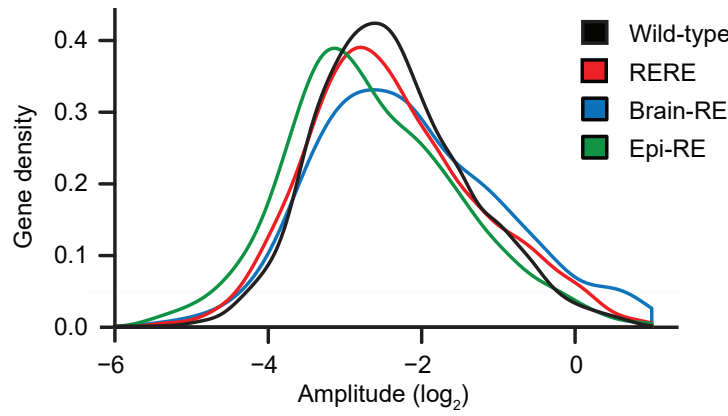

C
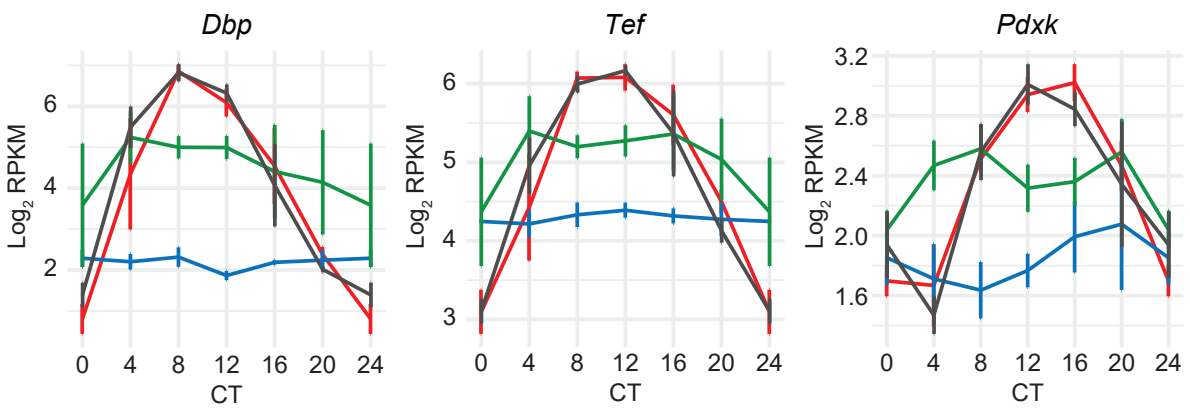

$\begin{array}{lllllll}0 & 4 & 8 & 12 & 16 & 20 & 24\end{array}$ CT

$\square$ Wild-type

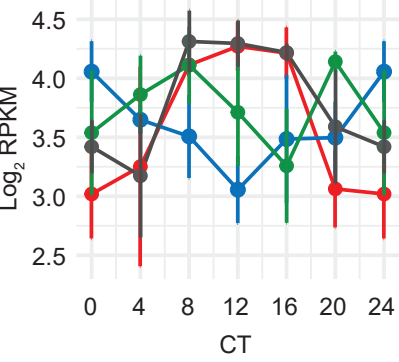

Mcm5

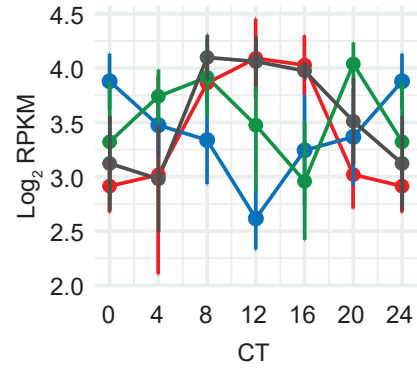

Pcna

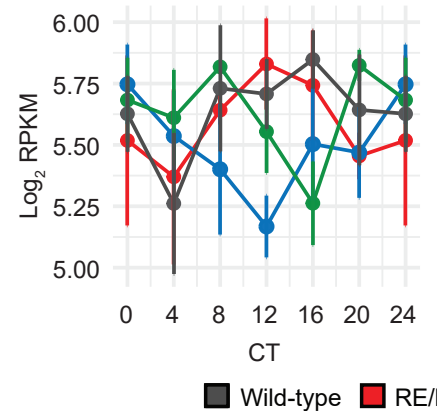

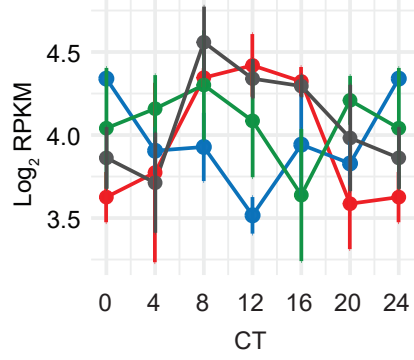

Mcm6

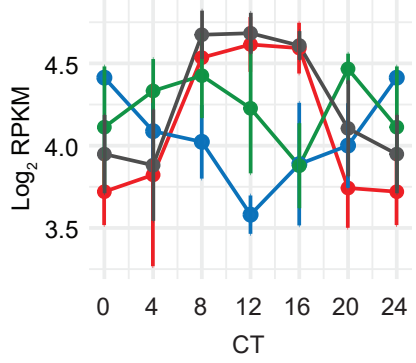

Pole

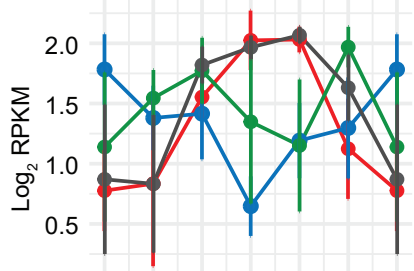

$\begin{array}{lllllll}0 & 4 & 8 & 12 & 16 & 20 & 24\end{array}$ CT
D

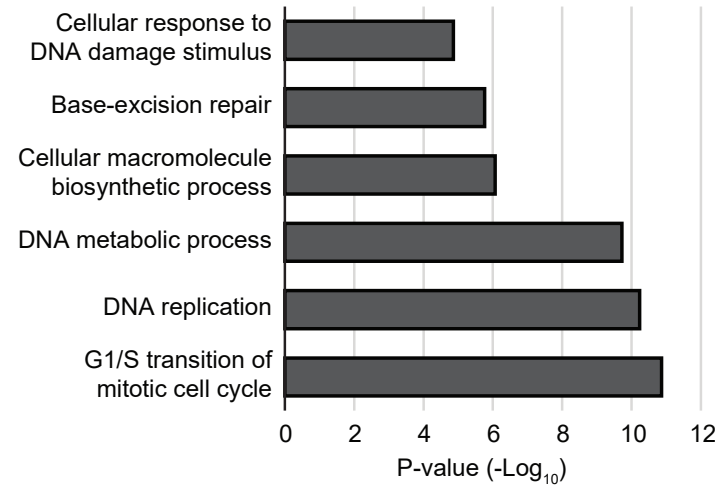

Brain-RE

E

Wild-type

RE/RE

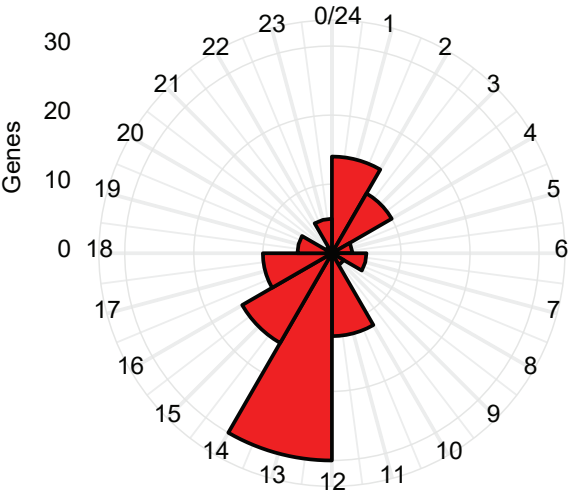

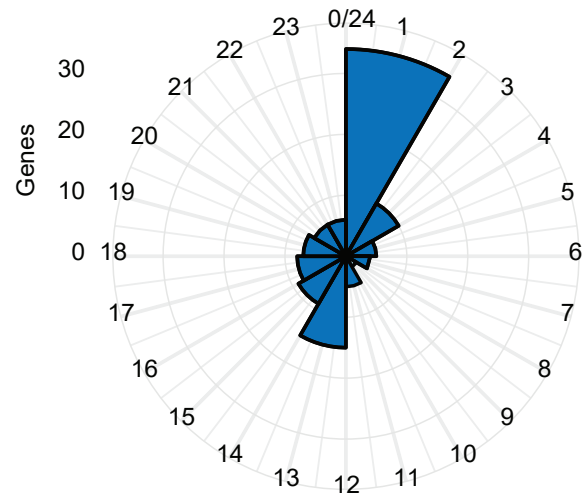

